\title{
Fluid-structure interaction between an incompressible, viscous 3D fluid and an elastic shell with nonlinear Koiter membrane energy
}

\author{
BORIS MUHA \\ Department of Mathematics, Faculty of Science, University of Zagreb, Croatia \\ E-mail: borism@math.hr \\ SUNČICA ČANIĆ \\ Department of Mathematics, University of Houston, Houston, Texas 77204-3476, USA \\ E-mail: canic@math.uh.edu
}

[Received 20 July 2014]

\begin{abstract}
The long-time existence of a weak solution is proved for a nonlinear, fluid-structure interaction (FSI) problem between an incompressible, viscous fluid and a semilinear cylindrical Koiter membrane shell with inertia. No axial symmetry is assumed in the problem. The fluid flow is driven by the timedependent dynamic pressure data prescribed at the inlet and outlet boundaries of the 3D cylindrical fluid domain. The fluid and the elastic structure are fully coupled via continuity of velocity and continuity of normal stresses. Global existence of a weak solution is proved as long as the lateral walls of the cylinder do not touch each other. The main novelty of the work is the nonlinearity in the structure model: the model accounts for the fully nonlinear Koiter membrane energy, supplemented with a small linear fourth-order derivative term modeling the bending rigidity of shells. The existence proof is constructive, and it is based on an operator splitting scheme. A version of this scheme can be implemented for the numerical simulation of the underlying FSI problem by extending the FSI solver, developed by the authors in [5], to include the nonlinearity in the structure model discussed in this manuscript.
\end{abstract}

2010 Mathematics Subject Classification: Primary 35Q35, 35R37, 74F10; Secondary 35A01, 35D30, 76D05, 74B20.

Keywords: Fluid-structure interaction, nonlinear moving boundary problem, nonlinear shell, existence of a weak solution.

\section{Introduction}

Fluid-structure interaction (FSI) problems arise in many physical, biological, and engineering problems. Perhaps the best known examples are aeroelasticity and biofluids. In biofluids, for example, a typical interaction between the fluid and soft tissue is nonlinear. An example is the interaction between blood flow and cardiovascular tissue (e.g., heart valves, or vascular tissue).

The mathematical analysis of such FSI problems remains to be a challenge due to the parabolichyperbolic nature of the problem, and due to the strongly nonlinear coupling in the case when the fluid and structure have comparable densities, which is the case in biofluidic applications. The nonlinearity in structural models brings additional difficulties to the underlying FSI problem, and only a few comprehensive results exist so far in this area, all by Shkoller et al. [7, 8, 14], except for the result in [9] which concerns nonlinearly forced linearly elastic structure (plate) interacting with an inviscid, incompressible fluid (see Section 3 for more details). The most closely related work to 


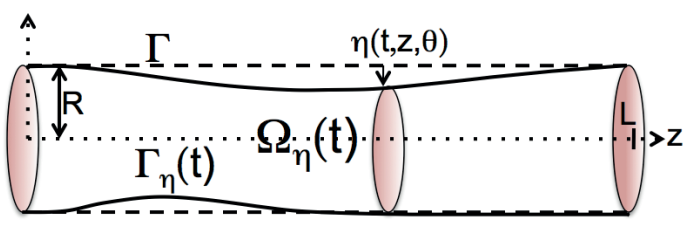

FIG. 1. Domain sketch

the one presented here is the work by Cheng and Shkoller [8] in which two scenarios involving a nonlinearly elastic Koiter shell interacting with a viscous, incompressible fluid were considered: a 2D fluid case for which the Koiter shell had non-zero inertia and arbitrary thickness, and a 3D fluid case for which the Koiter shell had zero inertia and its thickness had to be much smaller than the kinematic viscosity of the fluid. In both cases the shell was enclosing the fluid, and it served as a fluid domain boundary. Short time existence of a unique strong solution was obtained for both cases, and the analysis was performed entirely in the Lagrangian framework.

In the present work we prove the existence of a weak solution to a fluid-structure interaction problem between an 3D incompressible, viscous, Newtonian fluid and a semi-linear elastic cylindrical Koiter shell, globally in time until the lateral walls of the cylindrical fluid domain touch each other. The semi-linear elastic cylindrical Koiter shell model consists of a non-zero inertia term, the nonlinear terms corresponding to membrane energy, plus a higher-order linear term capturing regularizing effects due to the bending energy of shells, see (2.6). The fluid flow is driven by the time-dependent dynamic pressure data prescribed at the inlet and outlet boundaries of the fluid domain, see Figure 1. The fluid and structure are fully coupled via the kinematic and dynamic lateral boundary conditions describing continuity of velocity (the no-slip condition), and balance of contact forces at the fluid-structure interface, respectively. Because our fluid domain in not entirely enclosed by the elastic structure, we cannot employ a fully Lagrangian formulation of the problem as in [8]. Instead, we write the fluid problem in Eulerian formulation, and the structure problem in Lagrangian formulation, and use an Arbitrary Lagrangian-Eulerian mapping to map the coupled FSI problem onto a fixed domain. The resulting problem, however, has additional nonlinearities due to the motion of the fluid-structure interface, as expected. To prove the existence of a weak solution to this problem we semi-discretize the problem in time by using an operator splitting approach. By using the Lie operator splitting we separate the fluid from the structure problems and iterate between the two (once per time step) while satisfying the coupling conditions in an asynchronous way. The spitting is performed in a clever way so that the resulting coupled problem is stable in the corresponding energy norms, and compact in the sense that the semi-discretized approximate solutions converge strongly to a weak solution of the coupled FSI problem.

This approach is different from the one presented in [8]. The use of the Lie operator splitting approach to prove existence of solutions to FSI problems, first introduced by the authors in [36], was later used in $[4,5,21,23,34,36,38]$ to study various FSI problems involving linearly elastic structures. The main novelty of the present work is in adopting this robust approach to study FSI with a nonlinearly elastic structure of Koiter shell type. To deal with the nonlinearity in the structure problem we use the Schaefer's Fixed Point theorem [17, 42], which allowed us to prove the existence of a unique weak solution to the structure subproblem, and obtain energy estimates that mimic the energy of the continuous problem. The uniform energy estimates are a basis for the compactness argument, based on Simon's theorem [41], that provides strong convergence 
of approximate solutions to a weak solution of the FSI problem. Particularly interesting are the energy estimates that provide uniform bounds on the kinetic energy due to the motion of the fluid domain. These estimates lie at the interface between parabolic and hyperbolic regularity as they provide uniform bounds only on the half-order time derivatives of approximate solutions advancing in time. This is, however, sufficient for Simon's theorem to guarantee integral equicontinuity of the approximating sequences and ultimately the compactness and strong convergence to a weak solution.

While our long-time existence of a weak solution result presents an advancement in the theory of FSI problems involving nonlinearities in structure equations, the fact that the highest-order terms in our structure model are still linear, provide considerable help in the existence analysis of this FSI problem. Global existence involving a fully nonlinear (quasi linear) Koiter shell model with non-zero inertia remains to be a challenge in the theory of FSI problems in 3D.

\section{Model description}

We study the flow of an incompressible, viscous fluid in a three-dimensional cylindrical domain of reference length $L$, and reference radius $R$, see Figure 1 . We will be assuming that the lateral boundary of the cylinder is deformable and that its location is not known a priori, but is fully coupled to the motion of a viscous, incompressible fluid occupying the fluid domain. The lateral boundary is a thin, isotropic, homogeneous structure, whose dynamics is modeled by the nonlinear membrane equations containing an additional linear fourth-order term modeling bending rigidity of shells. For simplicity, we will be assuming that only the radial component of displacement is nonnegligible. This is, e.g., a common assumption in cardiovascular modeling [39]. In contrast with our earlier works, in this manuscript the structure equation incorporates nonlinear membrane effects, the problem is set in $3 D$, and the displacement of the structure is not assumed to be radially symmetric. Since neither the fluid flow, nor the displacement of the lateral boundary of the fluid domain will be required to satisfy the conditions of axial symmetry, the displacement $\eta$ will depend not only on the axial variable $z$ plus time, but also on the azimuthal variable $\theta$. Therefore, the radial displacement from the reference configuration will be denoted by $\eta(t, z, \theta)$. See Figure 1 .

Remark on notation We will be denoting by $(z, x, y)$ the Cartesian coordinates of points in $\mathbb{R}^{3}$ to describe the fluid flow equations, and by $(z, r, \theta)$ the corresponding cylindrical coordinates to describe the structure equations. A function $f$ given in Cartesian coordinates defines a function $\tilde{f}(z, r, \theta)=f(z, x, y)$ in cylindrical coordinates. Since no confusion is possible, to simplify notation we will omit the superscript ${ }^{\sim}$ and both functions, $f$ and $\tilde{f}$, will be denoted by $f$.

\subsection{The structure problem}

Consider a clamped cylindrical shell of thickness $h$, length $L$, and reference radius of the middle surface equal to $R$. This reference configuration, which we denote by $\Gamma$, see Figure 1, can be defined via the parameterization

$$
\varphi: \omega \rightarrow \mathbb{R}^{3}, \quad \varphi(z, \theta)=(R \cos \theta, R \sin \theta, z)^{t},
$$

where $\omega=(0, L) \times(0,2 \pi)$ and $R>0$. Therefore, the reference configuration is

$$
\Gamma=\left\{\mathbf{x}=(R \cos \theta, R \sin \theta, z) \in \mathbb{R}^{3}: \theta \in(0,2 \pi), z \in(0, L)\right\} .
$$


The associated covariant $A_{c}$ and contravariant $A^{c}$ metric tensors of this (undeformed) cylinder are given by:

$$
\mathbf{A}_{c}=\left(\begin{array}{cc}
1 & 0 \\
0 & R^{2}
\end{array}\right), \quad \mathbf{A}^{c}=\left(\begin{array}{cc}
1 & 0 \\
0 & \frac{1}{R^{2}}
\end{array}\right),
$$

and the area element along cylinder $\Gamma$ is $d S=\sqrt{a} d y:=\sqrt{\operatorname{det} A_{c}} d y=R d y$.

The displacement from the reference configuration $\Gamma$ of the deformed shell will be denoted by $\eta=\eta(t, z, \theta)=\left(\eta_{z}, \eta_{\theta}, \eta_{r}\right)$. We will be assuming that only the radial component of displacement is different from zero, and will be denoting that component by $\eta(t, z, \theta):=\eta_{r}(t, z, \theta)$, so that $\eta=\eta \mathbf{e}_{r}$, where $\mathbf{e}_{r}=\mathbf{e}_{r}(\theta)=(\cos \theta, \sin \theta, 0)^{t}$ is the unit vector in the radial direction.

The elastic properties of the cylindrical Koiter shell will be defined by the following elasticity tensor $Q$ :

$$
Q \mathbf{E}=\frac{4 \lambda \mu}{\lambda+2 \mu}\left(\mathbf{A}^{c} \cdot \mathbf{E}\right) \mathbf{A}^{c}+4 \mu \mathbf{A}^{c} \mathbf{E} \mathbf{A}^{c}, \quad \mathbf{E} \in \operatorname{Sym}\left(m_{2}\right)
$$

where $\mu$ and $\lambda$ are the Lamé coefficients. Using the following relationships between the Lamé constants and the Young's modulus of elasticity $E$ and Poisson ratio $\sigma$ :

$$
\frac{2 \mu \lambda}{\lambda+2 \mu}+2 \mu=4 \mu \frac{\lambda+\mu}{\lambda+2 \mu}=\frac{E}{1-\sigma^{2}}, \quad \frac{2 \mu \lambda}{\lambda+2 \mu}=4 \mu \frac{\lambda+\mu}{\lambda+2 \mu} \frac{1}{2} \frac{\lambda}{\lambda+\mu}=\frac{E}{1-\sigma^{2}} \sigma,
$$

the elasticity tensor $Q$ can also be written as:

$$
Q \mathbf{E}=\frac{2 E \sigma}{1-\sigma^{2}}\left(\mathbf{A}^{c} \cdot \mathbf{E}\right) \mathbf{A}^{c}+\frac{2 E}{1+\sigma} \mathbf{A}^{c} \mathbf{E} \mathbf{A}^{c}, \quad \mathbf{E} \in \operatorname{Sym}\left(m_{2}\right) .
$$

In our structure problem we will be accounting for the stretching of the middle surface, which is measured by the change of metric tensor (membrane effects), plus a small contribution coming from bending rigidity (shell effects). The membrane effect will be fully nonlinear, and corresponding to the true nonlinear Koiter membrane energy. The shell effect will be linear, and corresponding to a simple linearization of the higher-order terms is the Koiter shell bending energy. More precisely, by assuming only the radial component of displacement $\eta=\eta(t, r, \theta)$ to be different from zero, the full nonlinear change of metric tensor is given by the following:

$$
\frac{1}{2} \boldsymbol{G}(\eta)=\left(\begin{array}{cc}
\left(\partial_{z} \eta\right)^{2} & \partial_{z} \eta \partial_{\theta} \eta \\
\partial_{z} \eta \partial_{\theta} \eta & \eta(\eta+2 R)+\left(\partial_{\theta} \eta\right)^{2}
\end{array}\right) .
$$

This gives rise to the following nonlinear cylindrical Koiter membrane energy [10, 11, 26]:

$$
E_{e l}(\eta)=\frac{h}{4} \int_{\omega} \boldsymbol{Q} \boldsymbol{G}(\eta): \boldsymbol{G}(\eta) R d z d \theta
$$

As mentioned above, we add a small linear term modeling the bending rigidity of shells, so that the total elastic energy of the structure can be formally defined by

$$
E_{e l}(\eta)=\frac{h}{4} \int_{\omega} Q \boldsymbol{G}(\eta): \boldsymbol{G}(\eta) R d z d \theta+\varepsilon \int_{\omega}(\Delta \eta)^{2} R d z d \theta .
$$

Here $h$ is the thickness of the membrane shell, $\varepsilon>0$ is a bending rigidity parameter, and : denotes the scalar product

$$
\mathbf{A}: \mathbf{B}:=\operatorname{Tr}\left(\mathbf{A B} \mathbf{B}^{T}\right) \mathbf{A}, \mathbf{B} \in \mathbf{M}_{2}(\mathbb{R}) \cong \mathbb{R}^{4}
$$


The small term containing $\varepsilon$ has regularizing effects in the elastodynamics of the structure, providing important information about the solution via the energy estimates, presented below in Lemma 8.

The corresponding elastodynamic problem written in weak form then reads: Given a force $\mathbf{f}=$ $f \mathbf{e}_{r}$, with surface density $f$ (the radial component), find $\eta \in H_{0}^{2}(\omega)$ such that

$$
\rho_{K} h \int_{\omega} \partial_{t}^{2} \eta \xi R+\frac{h}{2} \int_{\omega} Q \boldsymbol{G}(\eta): \boldsymbol{G}^{\prime}(\eta) \xi R+\varepsilon \int_{\omega} \Delta \eta \Delta \xi R=\int_{\omega} f \xi R, \xi \in H_{0}^{2}(\omega),
$$

where $\varrho_{K}$ is the structure density, $h$ is the structure thickness, and $\mathbf{G}^{\prime}$ is the Gateux derivative of $\mathbf{G}$ :

$$
\mathbf{G}^{\prime}(\eta) \xi=\left(\begin{array}{cc}
\partial_{z} \eta \partial_{z} \xi & \frac{1}{2}\left(\partial_{z} \eta \partial_{\theta} \xi+\partial_{\theta} \eta \partial_{z} \xi\right) \\
\frac{1}{2}\left(\partial_{z} \eta \partial_{\theta} \xi+\partial_{\theta} \eta \partial_{z} \xi\right) & (R+\eta) \xi+\partial_{\theta} \eta \partial_{\theta} \xi
\end{array}\right) .
$$

To derive the corresponding differential form of the structure equations we first introduce a differential operator $\mathcal{L}_{m e m}$ corresponding to the Koiter membrane energy:

$$
\int_{\omega} \mathcal{L}_{m e m} \eta \xi R d z d \theta=\frac{h}{2} \int_{\omega} Q \boldsymbol{G}(\eta): \boldsymbol{G}^{\prime}(\eta) \xi R d z d \theta, \xi \in H_{0}^{2}(\omega),
$$

so that the above weak formulation can be written as

$$
\rho_{K} h \int_{\omega} \eta_{t t} \xi R+\int_{\omega} \mathcal{L}_{m e m} \eta \xi R+\varepsilon \int_{\omega} \Delta \eta \Delta \xi R=\int_{\omega} f \xi R, \xi \in H_{0}^{2}(\omega) .
$$

The corresponding differential formulation of our structure model (2.8) then reads:

$$
\varrho_{K} h \eta_{t t}+\frac{h}{2} £_{m e m} \eta+\varepsilon \Delta^{2} \eta=f \quad \text { in } \omega .
$$

Here, $\rho_{s}$ is the structure density, $h$ is the structure thickness, $\varepsilon$ is a regularizing bending coefficient, and $f$ is the force density in the radial (vertical) $\mathbf{e}_{r}$ direction acting on the structure. Operator $L_{m e m}$ in differential form is given by the following:

$$
\begin{aligned}
\mathcal{L}_{m e m} \eta= & \frac{h}{2}\left[\frac{R+\eta}{R^{2}}\left(\frac{E \sigma}{1-\sigma^{2}}\left(\partial_{z} \eta\right)^{2}+\frac{E}{\left(1-\sigma^{2}\right) R^{2}}\left(\eta(\eta+2 R)+\left(\partial_{\theta} \eta\right)^{2}\right)\right)\right. \\
& -\partial_{z}\left[\frac{E}{1-\sigma^{2}}\left(\partial_{z} \eta\right)^{3}+\frac{E \sigma}{\left(1-\sigma^{2}\right) R^{2}} \partial_{z} \eta\left(\eta(\eta+2 R)+\left(\partial_{\theta} \eta\right)^{2}\right)\right] \\
& -\frac{1}{R^{2}} \partial_{\theta}\left[\frac{E}{1-\sigma^{2}}\left(\partial_{z} \eta\right)^{2} \partial_{\theta} \eta+\frac{E \sigma}{\left(1-\sigma^{2}\right) R^{2}} \partial_{\theta} \eta\left(\eta(\eta+2 R)+\left(\partial_{\theta} \eta\right)^{2}\right)\right] \\
& \left.-\frac{E}{(1+\sigma) R^{2}}\left(\partial_{\theta}\left(\left(\partial_{z} \eta\right)^{2} \partial_{\theta} \eta\right)+\partial_{z}\left(\left(\partial_{\theta} \eta\right)^{2} \partial_{z} \eta\right)\right)\right]
\end{aligned}
$$

The partial differential equation (2.12) is supplemented with initial and boundary conditions. In this paper we will be assuming the clamped shell boundary condition:

$$
\eta=\frac{\partial \eta}{\partial \mathbf{n}}=0 \text { on } \partial \omega
$$




\subsection{The fluid problem}

The fluid domain, which depends on time and is not known a priori, will be denoted by

$$
\Omega_{\eta}(t)=\left\{(z, x, y) \in \mathbb{R}^{3}: \sqrt{x^{2}+y^{2}}<R+\eta(t, z, \theta), z \in(0, L)\right\},
$$

and the corresponding lateral boundary by

$$
\Gamma_{\eta}(t)=\left\{(z, x, y) \in \mathbb{R}^{3}: \sqrt{x^{2}+y^{2}}=R+\eta(t, z, \theta), z \in(0, L)\right\} .
$$

The inlet and outlet sections of the fluid domain boundary will be denoted by $\Gamma_{i n}=\{0\} \times(0, R)$, $\Gamma_{\text {out }}=\{L\} \times(0, R)$.

We are interested in studying a dynamic pressure-driven flow through $\Omega_{\eta}(t)$ of an incompressible, viscous fluid modeled by the Navier-Stokes equations which are given, in Cartesian coordinates, by:

$$
\left.\begin{array}{rl}
\rho_{f}\left(\partial_{t} \mathbf{u}+\mathbf{u} \cdot \nabla \mathbf{u}\right) & =\nabla \cdot \boldsymbol{\sigma}, \\
\nabla \cdot \mathbf{u} & =0,
\end{array}\right\} \text { in } \Omega_{\eta}(t), t \in(0, T)
$$

where $\rho_{f}$ denotes the fluid density, u fluid velocity, $p$ fluid pressure,

$$
\boldsymbol{\sigma}=-p \mathbf{I}+2 \mu_{F} \mathbf{D}(\mathbf{u})
$$

is the fluid Cauchy stress tensor, $\mu_{F}$ is the kinematic viscosity coefficient, and $\mathbf{D}(\mathbf{u})=\frac{1}{2}\left(\nabla \mathbf{u}+\nabla^{t} \mathbf{u}\right)$ is the symmetrized gradient of $\mathbf{u}$.

At the inlet and outlet boundaries we prescribe zero tangential velocity and dynamic pressure $p+\frac{\rho_{f}}{2}|u|^{2}$ (see, e.g., [12]):

$$
\left.\begin{array}{rl}
p+\frac{\rho_{f}}{2}|u|^{2} & =P_{\text {in/out }}(t), \\
\mathbf{u} \times \mathbf{e}_{z} & =0,
\end{array}\right\} \quad \text { on } \Gamma_{\text {in } / \text { out }},
$$

where $P_{\text {in } / \text { out }} \in L_{\text {loc }}^{2}(0, \infty)$ are given. Therefore the fluid flow is driven by a prescribed dynamic pressure drop, and the flow enters and leaves the fluid domain orthogonally to the inlet and outlet boundary.

The coupling between the fluid and structure is defined by two sets of boundary conditions satisfied at the lateral boundary $\Gamma_{\eta}(t)$. They are the kinematic and dynamic lateral boundary conditions describing continuity of velocity (the no-slip condition), and balance of contact forces (i.e., the Second Newton's Law of motion). Written in the Lagrangian framework, with $(z, \theta) \in \omega$, and $t \in(0, T)$, they read:

- The kinematic condition:

$$
\partial_{t} \eta(t, z, \theta) \mathbf{e}_{r}(\theta)=\boldsymbol{u}(t, z, R+\eta(t, z, \theta), \theta),
$$

where $\mathbf{e}_{r}(\theta)=(\cos \theta, \sin \theta, 0)^{t}$ is the unit vector in the radial direction.

- The dynamic condition:

$$
\rho_{K} h \partial_{t}^{2} \eta+\mathcal{L}_{m e m} \eta+\varepsilon \Delta^{2} \eta=-\left.J(t, z, \theta)(\sigma \mathbf{n})\right|_{(t, z, R+\eta(t, z, \theta))} \cdot \mathbf{e}_{r}(\theta),
$$


where $\mathcal{L}_{m e n}$ is defined by (2.10), and

$$
J(t, z, \theta)=\sqrt{\left(1+\partial_{z} \eta(t, z, \theta)^{2}\right)(R+\eta(t, z, \theta))^{2}+\partial_{\theta} \eta(t, z, \theta)^{2}}
$$

denotes the Jacobian of the composite function involving the transformation from Eulerian to Lagrangian coordinates and the transformation from cylindrical to Cartesian coordinates.

System (2.14)-(2.17) is supplemented with the following initial conditions:

$$
\mathbf{u}(0, .)=\mathbf{u}_{0}, \eta(0, .)=\eta_{0}, \partial_{t} \eta(0, .)=v_{0} .
$$

Additionally, we will be assuming that the initial data satisfies the following compatibility conditions:

$$
\begin{gathered}
\mathbf{u}_{0}\left(z, R+\eta_{0}(z), \theta\right) \cdot \mathbf{n}(z, \theta)=v_{0}(z, \theta) \mathbf{e}_{r}(\theta) \cdot \mathbf{n}(z, \theta), \quad z \in(0, L), \theta \in(0,2 \pi), \\
\eta_{0}=0, \quad \text { on } \partial \omega, \\
R+\eta_{0}(z, \theta)>0, \quad z \in[0, L], \theta \in(0,2 \pi) .
\end{gathered}
$$

Notice that the last condition requires that the initial displacement is such that the fluid domain has radius strictly greater than zero (i.e., the lateral boundary never collapses). This is an important condition which will be used at several places throughout this manuscript.

In summary, we study the following fluid-structure interaction problem:

Problem 1 Find $\mathbf{u}=\left(u_{z}(t, z, x, y), u_{x}(t, z, x, y), u_{y}(t, z, x, y)\right), p(t, z, x, y)$, and $\eta(t, z, \theta)$ such that

$$
\begin{aligned}
& \left.\begin{array}{rl}
\rho_{f}\left(\partial_{t} \mathbf{u}+(\mathbf{u} \cdot \nabla) \mathbf{u}\right) & =\nabla \cdot \boldsymbol{\sigma} \\
\nabla \cdot \mathbf{u} & =
\end{array}\right\} \text { in } \Omega_{\eta}(t), t \in(0, T), \\
& \left.\begin{array}{rl}
\mathbf{u} & =\partial_{t} \eta \mathbf{e}_{r}, \\
\rho_{K} h \partial_{t}^{2} \eta+\mathcal{L}_{m e m} \eta+\varepsilon \Delta^{2} \eta & =-J \boldsymbol{\sigma} \mathbf{n} \cdot \mathbf{e}_{r},
\end{array}\right\} \text { on }(0, T) \times \omega, \\
& \left.\begin{array}{rl}
p+\frac{\rho_{f}}{2}|u|^{2} & =P_{\text {in/out }}(t), \\
\mathbf{u} \times \mathbf{e}_{z} & =0,
\end{array}\right\} \text { on }(0, T) \times \Gamma_{\text {in } / \text { out }}, \\
& \left.\begin{array}{rl}
\mathbf{u}(0, .) & =\mathbf{u}_{0}, \\
\eta(0, .) & =\eta_{0}, \\
\partial_{t} \eta(0, .) & =v_{0},
\end{array}\right\} \text { at } t=0 .
\end{aligned}
$$

This is a nonlinear, moving-boundary problem in $3 \mathrm{D}$, which captures the full, two-way fluidstructure interaction coupling. The nonlinearity in the problem is represented by the quadratic term in the fluid equations, by the nonlinearity in the structure equation represented by the nonlinear membrane terms $L_{m e m}$, and by the nonlinear coupling between the fluid and structure defined at the moving (unknown) lateral boundary $\Gamma_{\eta}(t)$.

\section{A brief literature review}

Fluid-structure interaction problems have been actively studied for over 20 years now. Earlier works have focused on problems in which the coupling between the fluid and structure was calculated at a 
fixed fluid domain boundary, see [16], and [1, 2, 30], where an additional nonlinear coupling term was added and calculated at a fixed fluid interface. A study of well-posedness for FSI problems between an incompressible, viscous fluid and an elastic/viscoelastic structure with the coupling evaluated at a moving interface started with the result of daVeiga [3], where existence of a strong solution was obtained locally in time for an interaction between a $2 D$ fluid and a $1 D$ viscoelastic string, assuming periodic boundary conditions. This result was extended by Lequeurre in [32, 33], where the existence of a unique, local in time, strong solution for any data, and the existence of a global strong solution for small data, was proved in the case when the structure was modeled as a clamped viscoelastic beam.

D. Coutand and S. Shkoller proved existence, locally in time, of a unique, regular solution for an interaction between a viscous, incompressible fluid in $3 D$ and a $3 D$ structure, immersed in the fluid, where the structure was modeled by the equations of linear elasticity [13]. In the case when the structure (solid) is modeled by a linear wave equation, I. Kukavica et al. proved the existence, locally in time, of a strong solution, assuming lower regularity for the initial data [24, 27, 28]. A similar result for compressible flows can be found in [29]. In [40] the authors consider the FSI problem describing the motion of the elastic solid, described by equations of linear elasticity, inside an incompressible viscous fluid and prove existence and uniqueness of a strong solution. All the above mentioned existence results for strong solutions are local in time. Recently, in [25] a global existence result for small data was obtained for a similar moving boundary FSI problem but with additional interface and structure damping terms. We also mention that the works discussed in this paragraph were obtained in the context of Lagrangian coordinates, which were used for both the structure and fluid formulations.

In the context of weak solutions, the following results have been obtained. Continuous dependence of weak solutions on initial data for a fluid-structure interaction problem with a free boundary type coupling condition was studied in [22]. Existence of a weak solution for a FSI problem between a $3 D$ incompressible, viscous fluid and a $2 D$ viscoelastic plate was shown by Chambolle et al. in [6], while Grandmont improved this result in [19] to hold for a $2 D$ elastic plate. These results were extended to a more general geometry in [31], and to a non-Newtonian shear dependent fluid in [34]. In these works existence of a weak solution was proved for as long as the elastic boundary does not touch "the bottom" (rigid) portion of the fluid domain boundary.

Muha and Canić recently proved the existence of weak solutions to a class of FSI problems modeling the flow of an incompressible, viscous, Newtonian fluid flowing through a 2D cylinder whose lateral wall was modeled by either the linearly viscoelastic, or by the linearly elastic Koiter shell equations [36], assuming nonlinear coupling at the deformed fluid-structure interface. The fluid flow boundary conditions were not periodic, but rather, the flow was driven by the dynamic pressure drop data. The main novelty was in the design of a novel methodology for proving the existence of a weak solution to an entire class of FSI problems: a constructive existence proof was presented based on the Lie operator splitting scheme, which was used for the numerical simulation of several FSI problems [4, 5, 21, 23, 34, 36]. This methodology was recently extended to a FSI problem with two structural layers (composite structures) in [37], and to a $3 D$ fluid case coupled to the elastodynamics of a linearly elastic Koiter shell in [38].

All the works mentioned above consider FSI problems involving linearly elastic structures. Despite an enormous interest in FSI problems with nonlinear structures arising in various applications, there are only a few well-posedness results in this area. They come from the group of Shkoller et al. where short-time existence of a unique, regular solution was proved for several 
different problems, all in the context of global Lagrangian formulation [7, 8, 14]. In particular, in [14] the authors study a $3 D$ FSI problem between an incompressible, viscous, Newtonian fluid and a nonlinear, large-displacement elastic solid, modeled by the St. Venant-Kirchhoff constitutive law. By using parabolic regularization with a particular artificial viscosity, the authors prove the existence of a unique (locally in time) regular solution in Sobolev spaces. In [7, 8] FSI problems between an incompressible, viscous Newtonian fluid and thin nonlinear shells were studied. The work in [7] considers a biofluid shell whose bending energy is modeled by the Willmore function in $3 D$, while the work in [8] considers the nonlinear Koiter shell model, both in $2 D$ and $3 D$. In both works the existence of a unique (locally in time) strong solution was obtained. However, in both of those works, whenever the $3 D$ fluid case was considered, the corresponding structure problem was quasi-static, i.e., the structural problem had zero inertia. Furthermore, in the case of the Koiter shell problem in $3 D$, the existence results was obtained under an additional assumption that the shell thickness is much smaller than the kinematic viscosity of the fluid.

The present manuscript is a first step towards proving the existence of a global weak solution for a 3D FSI problem between an incompressible, viscous fluid and a thin nonlinearly elastic shell. By global we mean that a solution exists until the structure, which serves as a portion of the fluid boundary, touches another piece of the fluid boundary. As described earlier in Section 2, our structural model has non-zero inertia, a contribution from the nonlinear Koiter membrane energy, and a small regularizing linear fourth-order term describing the bending rigidity of shells. The method of proof is different from the methods developed in [7, 8, 14]. It is a nontrivial extension of our earlier methodology, which is based on semi-discretization via operator splitting. The main novelty is in dealing with the nonlinear terms corresponding to the membrane energy. This requires a careful discretization in time of the nonlinear membrane terms in order to obtain uniform energy estimates, and the use of the Schaefer's fixed point theorem. Moreover, there are additional difficulties due to the low regularity of solutions, including making sense of the trace of the fluid velocity at the fluid-structure interface, and using the appropriate compactness results. By showing that the approximating sequences converge to a weak solution to the underlying problem, we effectively show that the corresponding numerical scheme, which can be designed from the techniques presented in this manuscript, is stable and converges to a weak solution of this FSI problem.

\subsection{The energy of the problem}

Assuming that a solution to Problem 1 exists and is sufficiently regular, we formally derive an energy inequality for the coupled FSI problem. To simplify notation, we introduce the following energy norm defined by the membrane effects:

$$
\|\eta\|_{\gamma}^{4}:=\int_{\omega} \boldsymbol{Q} \boldsymbol{G}(\eta): \boldsymbol{G}(\eta) R d z d \theta,
$$

which can be written explicitly by using formulas (2.3) and (2.4) as:

$$
\begin{aligned}
\|\eta\|_{\gamma}^{4}=\int_{\omega} \frac{2 E R \sigma}{1-\sigma^{2}} & \left(\left(\partial_{z} \eta\right)^{2}+\frac{1}{R}\left(\eta(\eta+R)+\left(\partial_{\theta} \eta\right)^{2}\right)\right)^{2} d z d \theta \\
& +\int_{\omega} \frac{2 E R}{1+\sigma}\left(\left(\partial_{z} \eta\right)^{4}+\frac{2}{R}\left(\partial_{z} \eta\right)^{2}\left(\partial_{\theta} \eta\right)^{2}+\frac{1}{R^{2}}\left(\eta(\eta+R)+\left(\partial_{\theta} \eta\right)^{2}\right)^{2}\right) d z d \theta .
\end{aligned}
$$


It is easy to show that the norm $\|\cdot\|_{\gamma}$ is equivalent to the standard $W^{1,4}(\omega)$ norm. The following Proposition states that the kinetic and elastic energy of the coupled FSI problem are bounded by a constant, which depends only on the prescribed inlet and outlet data.

PROPOSITION 1 Assuming sufficient regularity, solutions of Problem 1 satisfy the following energy estimate:

$$
\frac{d}{d t}\left(E_{k i n}(t)+E_{e l}(t)\right)+D(t) \leqslant C\left(P_{\text {in }}(t), P_{\text {out }}(t)\right),
$$

where

$$
\begin{gathered}
E_{k i n}(t):=\frac{1}{2}\left(\rho_{f}\|\mathbf{u}\|_{L^{2}\left(\Omega_{F}(t)\right)}^{2}+\rho_{K} h\left\|\partial_{t} \eta\right\|_{L^{2}(\Gamma)}^{2}\right), \\
E_{e l}(t):=\frac{h}{4}\|\eta\|_{\gamma}^{4}+\varepsilon\|\Delta \eta\|_{L^{2}(\omega)}^{2},
\end{gathered}
$$

denote the kinetic and elastic energy of the coupled problem, respectively, and term $D(t)$ captures viscous dissipation in the fluid:

$$
D(t):=\mu_{F}\|\mathbf{D}(\mathbf{u})\|_{L^{2}\left(\Omega_{F}(t)\right)}^{2} .
$$

The constant $C\left(P_{\text {in }}(t), P_{\text {out }}(t)\right)$ depends only on the inlet and outlet pressure data, which are both functions of time.

The proof of inequality (3.2) is standard (see, e.g., [36]). Later in this manuscript it will be rigorously shown that the weak solutions constructed in this work satisfy the above energy estimate. Notice that the boundedness of energy implies boundedness of the $H^{2}(\omega)$ norm of the solution. This is due to the regularizing term $\varepsilon\|\Delta \eta\|_{L^{2}(\Omega)}^{2}$, and is a consequence of the standard elliptic regularity theory on polygonal domains (see, e.g., [20], Thm 2.2.3).

\section{Weak formulation}

\subsection{ALE mapping}

To prove the existence of a weak solution to Problem 1 it is convenient to map Problem 1 onto a fixed domain $\Omega$. We choose $\Omega$ to be the reference cylinder of radius $R$ and length $L$ :

$$
\Omega=\left\{(z, x, y): z \in(0, L), x^{2}+y^{2}<R\right\} .
$$

We follow the approach typical of numerical methods for fluid-structure interaction problems and map our fluid domain $\Omega(t)$ onto $\Omega$ by using an Arbitrary Lagrangian-Eulerian (ALE) mapping $[5,15,21,39]$. We remark here that in our problem it is not convenient to use the Lagrangian formulation of the fluid sub-problem, as is done in e.g., [8, 14, 27], since, in our problem, the fluid domain consists of a fixed, control volume of a cylinder, which does not follow Largangian flow.

We begin by defining a family of ALE mappings $A_{\eta}$ parameterized by $\eta$ :

$$
\left.A_{\eta}(t): \Omega \rightarrow \Omega_{\eta}(t), \quad A_{\eta}(t)(\tilde{z}, \tilde{r}, \tilde{\theta}):=\left(\begin{array}{c}
\tilde{z} \\
\tilde{\theta} \\
(t, \tilde{z}, \tilde{\theta})
\end{array}\right) \tilde{r}\right), \quad(\tilde{z}, \tilde{r}, \tilde{\theta}) \in \Omega,
$$

where $(\tilde{z}, \tilde{r}, \tilde{\theta})$ denote the cylindrical coordinates in the reference domain $\Omega$. See Figure 2 . Since we work with the Navier-Stokes equations written in Cartesian coordinates, it is useful to write an 


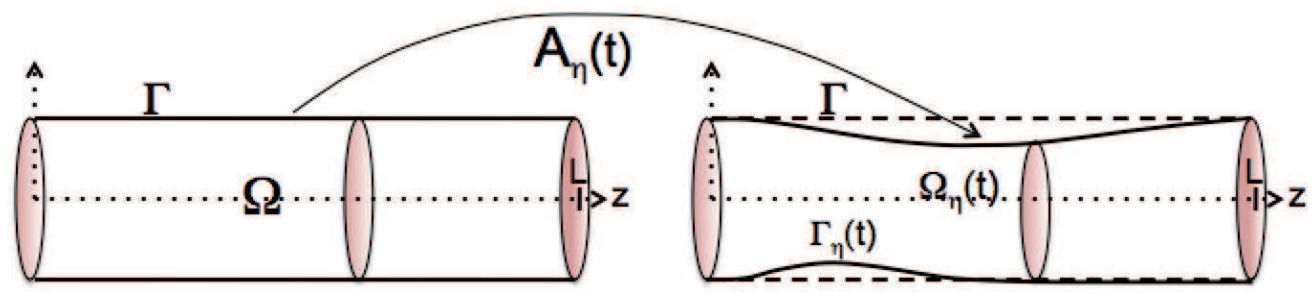

FIG. 2. ALE mapping

explicit form of the ALE mapping $A_{\eta}$ in Cartesian coordinates as well:

$$
A_{\eta}(t)(\tilde{z}, \tilde{x}, \tilde{y}):=\left(\begin{array}{c}
\tilde{z} \\
(R+\eta(t, \tilde{z}, \tilde{\theta})) \tilde{x} \\
(R+\eta(t, \tilde{z}, \tilde{\theta})) \tilde{y}
\end{array}\right), \quad(\tilde{z}, \tilde{x}, \tilde{y}) \in \Omega .
$$

The mapping $A_{\eta}(t)$ is a bijection, and its Jacobian is given by

$$
\left|\operatorname{det} \nabla A_{\eta}(t)\right|=(R+\eta(t, \tilde{z}, \tilde{\theta}))^{2} .
$$

Composite functions with the ALE mapping will be denoted by

$$
\mathbf{u}^{\eta}(t, .)=\mathbf{u}(t, .) \circ A_{\eta}(t) \quad \text { and } \quad p^{\eta}(t, .)=p(t, .) \circ A_{\eta}(t) .
$$

Derivatives of composite functions satisfy:

$$
\nabla \mathbf{u}=\nabla \mathbf{u}^{\eta}\left(\nabla A_{\eta}\right)^{-1}=: \nabla^{\eta} \mathbf{u}^{\eta}, \quad \partial_{t} \mathbf{u}=\partial_{t} \mathbf{u}^{\eta}-\left(\mathbf{w}^{\eta} \cdot \nabla^{\eta}\right) \mathbf{u}^{\eta},
$$

where the ALE domain velocity, $\mathbf{w}^{\eta}$, is given by:

$$
\mathbf{w}^{\eta}=\partial_{t} \eta\left(\begin{array}{c}
0 \\
\tilde{x} \\
\tilde{y}
\end{array}\right) .
$$

The following notation will also be useful:

$$
\boldsymbol{\sigma}^{\eta}=-p^{\eta} \mathbf{I}+2 \mu_{F} \mathbf{D}^{\eta}\left(\mathbf{u}^{\eta}\right), \quad \mathbf{D}^{\eta}\left(\mathbf{u}^{\eta}\right)=\frac{1}{2}\left(\nabla^{\eta} \mathbf{u}^{\eta}+\left(\nabla^{\eta}\right)^{\tau} \mathbf{u}^{\eta}\right) .
$$

We are now ready to rewrite Problem 1 mapped onto domain $\Omega$. However, before we do that, we will make one more important step in our strategy to prove the existence of a weak solution to Problem 1. Namely, we would like to "solve" the coupled FSI problem by approximating the problem using the time-discretization via operator splitting, and then prove that the solution to the semi-discrete problem converges to a weak solution of the continuous problem, as the time-discretization step tends to zero. To perform the time discretization via operator splitting, which will be described in the next section, we need to write our FSI problem as a first-order system in time. This will be done by replacing the second-order time-derivative of $\eta$, with the first-order time-derivative of the structure velocity. To do this, we further notice that in the coupled FSI problem, the kinematic 
coupling condition (2.16) implies that the structure velocity is equal to the normal trace of the fluid velocity on $\Gamma_{\eta}(t)$. Thus, we will introduce a new variable, $v$, to denote this trace, and will replace $\partial_{t} \eta$ by $v$ everywhere in the structure equation. This has deep consequences both for the existence proof presented in this manuscript, as well as for the proof of stability of the underlying numerical scheme, presented in [44], as it enforces the kinematic coupling condition implicitly in all the steps of the scheme.

Thus, Problem 1 can be reformulated in the ALE framework, on the reference domain $\Omega$, and written as a first-order system in time, in the following way:

Problem 2 Find $\mathbf{u}^{\eta}(t, \tilde{z}, \tilde{x}, \tilde{y}), p^{\eta}(t, \tilde{z}, \tilde{x}, \tilde{y}), \eta(t, \tilde{z}, \tilde{\theta})$, and $v(t, \tilde{z}, \tilde{\theta})$ such that

$$
\begin{aligned}
& \left.\begin{array}{rl}
\rho_{f}\left(\partial_{t} \mathbf{u}^{\eta}+\left(\left(\mathbf{u}^{\eta}-\mathbf{w}^{\eta}\right) \cdot \nabla^{\eta}\right) \mathbf{u}^{\eta}\right) & =\nabla^{\eta} \cdot \boldsymbol{\sigma}^{\eta}, \\
\nabla^{\eta} \cdot \mathbf{u}^{\eta} & =0,
\end{array}\right\} \text { in }(0, T) \times \Omega, \\
& \left.\begin{array}{rl}
p+\frac{\rho_{f}}{2}\left|u^{\eta}\right|^{2} & =P_{\text {in/out }}(t), \\
\mathbf{u}^{\eta} \times \mathbf{e}_{z} & =0,
\end{array}\right\} \text { on }(0, T) \times \Gamma_{\text {in } / \text { out }}, \\
& \left.\begin{array}{rl}
\mathbf{u}^{\eta} & =v \mathbf{e}_{r}, \\
\partial_{t} \eta & =v, \\
\rho_{K} h \partial_{t} v+\mathcal{L}_{m e m} \eta+\varepsilon \Delta^{2} \eta & =-J \boldsymbol{\sigma}^{\eta} \mathbf{n} \cdot \mathbf{e}_{r}
\end{array}\right\} \text { on }(0, T) \times \omega, \\
& \mathbf{u}^{\eta}(0, .)=\mathbf{u}_{0}, \eta(0, .)=\eta_{0}, v(0, .)=v_{0}, \quad \text { at } \quad t=0 .
\end{aligned}
$$

To simplify notation, in the remainder of the manuscript we drop the superscript $\eta$ in $\mathbf{u}^{\eta}$ whenever there is no chance of confusion.

\subsection{Weakformulation}

To define weak solutions of the moving-boundary problem (4.7)-(4.10) defined on $\Omega$, we introduce the following notation and function spaces.

For the fluid velocity we would like to work with the classical function space associated with weak solutions of the Navier-Stokes equations. This, however, requires some additional consideration. Namely, since the fluid domain is also an unknown in the problem, we cannot assume a priori any smoothness that is not consistent with the energy estimates, and so the fluid domain boundary may not even be Lipschitz. Indeed, from the energy inequality (3.2) we only have $\eta \in H^{2}(\omega)$, and from Sobolev embeddings, by taking into account that we are working in $\mathbb{R}^{3}$, we get that $\eta \in C^{0, \mu}(\omega), \mu<1$. Therefore, the energy estimates imply that $\Omega_{\eta}(t)$ is not necessarily a Lipschitz domain. However, $\Omega_{\eta}(t)$ is locally a sub-graph of a Hölder continuous function. In that case one can define the "Lagrangian" trace

$$
\begin{aligned}
& \gamma_{\Gamma(t)}: C^{1}\left(\overline{\Omega_{\eta}(t)}\right) \rightarrow C(\omega), \\
& \gamma_{\Gamma(t)}: v \mapsto v(t, z, R+\eta(t, z, \theta), \theta) .
\end{aligned}
$$

It was shown in $[6,19,35]$ that the trace operator $\gamma_{\Gamma(t)}$ can be extended by continuity to a linear operator from $H^{1}\left(\Omega_{\eta}(t)\right)$ to $H^{s}(\omega), 0 \leqslant s<\frac{1}{2}$. For a precise statement of the results about 
"Lagrangian" trace see [35]. Now, we can define the velocity solution space defined on the moving domain in the following way:

$$
\begin{aligned}
& V_{F}(t)=\left\{\mathbf{u}=\left(u_{z}, u_{x}, u_{y}\right) \in C^{1}\left(\overline{\Omega_{\eta}(t)}\right)^{3}: \nabla \cdot \mathbf{u}=0,\right. \\
& \left.\quad \mathbf{u} \times \mathbf{e}_{r}=0 \text { on } \Gamma(t), \mathbf{u} \times \mathbf{e}_{z}=0 \text { on } \Gamma_{\text {in } / \text { out }}\right\}, \\
& V_{F}(t)={\overline{V_{F}(t)}}^{H^{1}\left(\Omega_{\eta}(t)\right) .} .
\end{aligned}
$$

Using the fact that $\Omega_{\eta}(t)$ is locally a sub-graph of a Hölder continuous function we can get the following characterization of the velocity solution space $v_{F}(t)$ (see $\left.[6,19]\right)$ :

$$
\begin{aligned}
V_{F}(t)=\left\{\mathbf{u}=\left(u_{z}, u_{x}, u_{y}\right)\right. & \in H^{1}\left(\Omega_{\eta}(t)\right)^{3}: \nabla \cdot \mathbf{u}=0, \\
& \left.\mathbf{u} \times \mathbf{e}_{r}=0 \text { on } \Gamma(t), \mathbf{u} \times \mathbf{e}_{z}=0 \text { on } \Gamma_{\text {in } / \text { out }}\right\} .
\end{aligned}
$$

Before defining the fluid velocity space defined on the fixed, reference domain $\Omega$, it is important to point out that the transformed fluid velocity $\mathbf{u}^{\eta}$ is not divergence-free anymore. Rather, it satisfies the transformed divergence-free condition $\nabla^{\eta} \cdot \mathbf{u}^{\eta}=0$. Furthermore, since $\eta$ is not Lipschitz, the ALE mapping is not necessarily a Lipschitz function either, and, as a result, $\mathbf{u}^{\eta}$ is not necessarily in $H^{1}(\Omega)$. Therefore, we need to redefine the function spaces for the fluid velocity by introducing

$$
V_{F}^{\eta}=\left\{\mathbf{u}^{\eta}: \mathbf{u} \in V_{F}(t)\right\},
$$

where $\mathbf{u}^{\eta}$ is defined in (4.4). Under the assumption $R+\eta(t, z, \theta)>0, z \in[0, L]$, we can define a scalar product on $V_{F}^{\eta}$ in the following way:

$$
\begin{aligned}
\left(\mathbf{u}^{\eta}, \mathbf{v}^{\eta}\right)_{v_{F}^{\eta}} & =\int_{\Omega}(R+\eta)^{2}\left(\mathbf{u}^{\eta} \cdot \mathbf{v}^{\eta}+\nabla^{\eta} \mathbf{u}^{\eta}: \nabla^{\eta} \mathbf{v}^{\eta}\right) \\
& =\int_{\Omega_{\eta}(t)} \mathbf{u} \cdot \mathbf{v}+\nabla \mathbf{u}: \nabla \mathbf{v}=(\mathbf{u}, \mathbf{v})_{H^{1}\left(\Omega_{\eta}(t)\right)} .
\end{aligned}
$$

Therefore, $\mathbf{u} \mapsto \mathbf{u}^{\eta}$ is an isometric isomorphism between $V_{F}(t)$ and $V_{F}^{\eta}$, and so $V_{F}^{\eta}$ is also a Hilbert space.

The function space associated with the Koiter shell equations is standard:

$$
v_{K}=H_{0}^{2}(\omega) .
$$

From this point on we will be working with the FSI problem mapped via the ALE mapping onto the fixed, reference domain $\Omega$. Motivated by the energy inequality we define the corresponding evolution spaces for the FSI problem defined on $\Omega$ :

$$
\begin{aligned}
& W_{F}^{\eta}(0, T)=L^{\infty}\left(0, T ; L^{2}(\Omega)\right) \cap L^{2}\left(0, T ; V_{F}^{\eta}\right), \\
& W_{K}(0, T)=W^{1, \infty}\left(0, T ; L^{2}(\omega)\right) \cap L^{2}\left(0, T ; H_{0}^{2}(\omega)\right),
\end{aligned}
$$

The corresponding solution and test spaces are defined by:

$$
\begin{aligned}
& W^{\eta}(0, T)=\left\{(\mathbf{u}, \eta) \in W_{F}^{\eta}(0, T) \times W_{K}(0, T): \mathbf{u}_{\mid r=R}=\partial_{t} \eta \mathbf{e}_{r},\right\} . \\
& Q^{\eta}(0, T)=\left\{(\mathbf{q}, \xi) \in C_{c}^{1}\left([0, T) ; V_{F}^{\eta} \times V_{K}\right): \mathbf{q} \mid r=R=\xi \mathbf{e}_{r}\right\} .
\end{aligned}
$$


We will be using $b^{\eta}$ to denote the following trilinear form corresponding to the (symmetrized) nonlinear advection term in the Navier-Stokes equations in the fixed, reference domain:

$$
b^{\eta}(\mathbf{u}, \mathbf{u}, \mathbf{q}):=\frac{1}{2} \int_{\Omega}(R+\eta)^{2}\left(\left(\mathbf{u}-\mathbf{w}^{\eta}\right) \cdot \nabla^{\eta}\right) \mathbf{u} \cdot \mathbf{q}-\frac{1}{2} \int_{\Omega}(R+\eta)^{2}\left(\left(\mathbf{u}-\mathbf{w}^{\eta}\right) \cdot \nabla^{\eta}\right) \mathbf{q} \cdot \mathbf{u} .
$$

Finally, we define a linear functional which associates the inlet and outlet dynamic pressure boundary data to a test function $\mathbf{v}$ in the following way:

$$
\langle F(t), \mathbf{v}\rangle_{\Gamma_{\text {in } / \text { out }}}=P_{\text {in }}(t) \int_{\Gamma_{\text {in }}} v_{z}-P_{\text {out }}(t) \int_{\Gamma_{\text {out }}} v_{z}
$$

Definition 2 We say that $(\mathbf{u}, \eta) \in W^{\eta}(0, T)$ is a weak solution of problem (4.7)-(4.10) defined on the reference domain $\Omega$, if for all $(\mathbf{q}, \psi) \in Q^{\eta}(0, T)$ the following equality holds:

$$
\begin{gathered}
-\rho_{f}\left(\int_{0}^{T} \int_{\Omega}(R+\eta)^{2} \mathbf{u} \cdot \partial_{t} \mathbf{q}+\int_{0}^{T} b^{\eta}(\mathbf{u}, \mathbf{u}, \mathbf{q})\right)+2 \mu_{F} \int_{0}^{T} \int_{\Omega}(R+\eta)^{2} \mathbf{D}^{\eta}(\mathbf{u}): \mathbf{D}^{\eta}(\mathbf{q}) \\
-\rho_{f} \int_{0}^{T} \int_{\Omega}(R+\eta)\left(\partial_{t} \eta\right) \mathbf{u} \cdot \mathbf{q}-R \rho_{K} h \int_{0}^{T} \int_{\omega} \partial_{t} \eta \partial_{t} \xi+\frac{R h}{2} \int_{0}^{T} \int_{\omega} Q \boldsymbol{G}(\eta): \boldsymbol{G}^{\prime}(\eta) \xi \\
\quad+\varepsilon R \int_{0}^{T} \int_{\omega} \Delta \eta \Delta \xi \\
=\int_{0}^{T}\langle F(t), \mathbf{q}\rangle_{\Gamma_{\text {in } / \text { out }}}+\int_{\Omega_{\eta}}\left(R+\eta_{0}\right)^{2} \mathbf{u}_{0} \cdot \mathbf{q}(0)+\int_{\omega} v_{0} \xi(0) .
\end{gathered}
$$

The weak formulation is obtained in the standard way by multiplying the PDE by a test function and integrating by parts. More details can be found in [36], Section 4 and [38], Section 3.2.

\section{The operator splitting scheme}

We semidiscretize the coupled FSI problem in time by performing the time discretization via operator splitting. At each time step a couple of semi-discretized problems will be solved, one for the fluid and one for the structure, with certain initial and boundary conditions reflecting the coupling between the two. The operator splitting will be performed in a clever way so that sequences of approximating solutions satisfy uniform energy estimates which mimic the energy of the continuous problem. To perform the desired splitting, we employ the Lie splitting strategy, also known as the Marchuk-Yanenko splitting scheme.

\section{$5.1 \quad$ Lie splitting}

For a given time interval $(0, T)$, introduce $N \in \mathbb{N}, \Delta t=T / N$ and $t_{n}=n \Delta t$. Consider the following initial-value problem:

$$
\frac{d \phi}{d t}+A \phi=0 \quad \text { in }(0, T), \quad \phi(0)=\phi_{0},
$$


where $A$ is an operator defined on a Hilbert space, and $A$ can be written as $A=A_{1}+A_{2}$. Set $\phi^{0}=\phi_{0}$, and, for $n=0, \ldots, N-1$ and $i=1,2$, compute $\phi^{n+\frac{i}{2}}$ by solving

$$
\left.\begin{array}{rl}
\frac{d}{d t} \phi_{i}+A_{i} \phi_{i} & =0 \\
\phi_{i}\left(t_{n}\right) & =\phi^{n+\frac{i-1}{2}}
\end{array}\right\} \quad \text { in }\left(t_{n}, t_{n+1}\right),
$$

and then set $\phi^{n+\frac{i}{2}}=\phi_{i}\left(t_{n+1}\right)$, for $i=1,2$. It can be shown that this method is first-order accurate in time, see e.g., [18].

\subsection{Approximate solutions}

We apply this approach to split Problem 2 into the fluid and structure sub-problems. During this procedure the structure equation (4.9) will be split into two parts: everything involving only the normal trace $v$ of the fluid velocity on $\Gamma_{\eta}(t)$ will be used in the boundary condition for the fluid subproblem (Problem A2), and the remaining purely elastodynamics part of the structure equation will be used in the structure subproblem (Problem A1).

As mentioned above, the Lie splitting defines a time step, which we denoted by $\Delta t$, and a number of time sub-intervals $N \in \mathbb{N}$, so that

$$
(0, T)=\cup_{n=0}^{N-1}\left(t^{n}, t^{n+1}\right), \quad t^{n}=n \Delta t, n=0, \ldots, N-1 .
$$

For every subdivision containing $N \in \mathbb{N}$ sub-intervals, we recursively define the vector of unknown approximate solutions

$$
\mathbf{X}_{N}^{n+\frac{i}{2}}=\left(\begin{array}{c}
\mathbf{u}_{N}^{n+\frac{i}{2}} \\
n+\frac{i}{2} \\
v_{N}^{n+\frac{i}{2}} \\
\eta_{N}
\end{array}\right), n=0,1, \ldots, N-1, i=1,2,
$$

where $i=1,2$ denotes the solution of sub-problem A1 or A2, respectively. The initial condition will be denoted by

$$
\mathbf{X}^{0}=\left(\begin{array}{c}
\mathbf{u}_{0} \\
v_{0} \\
\eta_{0}
\end{array}\right)
$$

The semi-discretization and the splitting of the problem will be performed in such a way that the discrete version of the energy inequality (3.2) is preserved at every time step. This is a crucial ingredient for the existence proof.

We define the semi-discrete versions of the kinetic and elastic energy, originally defined in (3.3), and of dissipation, originally defined in (3.4), by the following:

$$
\begin{gathered}
E_{N}^{n+\frac{i}{2}}=\frac{1}{2}\left(\rho_{f} \int_{\Omega}\left(R+\eta^{n-1+i}\right)^{2}\left|\mathbf{u}_{N}^{n+\frac{i}{2}}\right|^{2}+\rho_{s} h\left\|v_{N}^{n+\frac{i}{2}}\right\|_{L^{2}(\omega)}^{2}\right. \\
\left.+\frac{h}{2}\left\|\eta_{N}^{n+\frac{i}{2}}\right\|_{\gamma}^{4}+\varepsilon\left\|\Delta \eta_{N}^{n+\frac{i}{2}}\right\|_{L^{2}(\omega)}^{2}\right), \\
D_{N}^{n+1}=\Delta t \mu_{F} \int_{\Omega}\left(R+\eta^{n}\right)^{2}\left|D^{\eta^{n}}\left(\mathbf{u}_{N}^{n+1}\right)\right|^{2}, n=0, \ldots, N-1, i=0,1 .
\end{gathered}
$$


Notice how the presence of the nonlinear membrane terms in the Koiter shell model gives rise to the new norm $\|\cdot\|_{\gamma}$ which appears in (5.2) to the 4-th power. This term implies higher regularity estimates than what we would have gotten in the linear Koiter membrane case.

Throughout the rest of this section, we fix the time step $\Delta t$, i.e., we keep $N \in \mathbb{N}$ fixed, and study the semi-discretized sub-problems defined by the Lie splitting. To simplify notation, we will omit the subscript $N$ and write $\left(\mathbf{u}^{n+\frac{i}{2}}, v^{n+\frac{i}{2}}, \eta^{n+\frac{i}{2}}\right)$ instead of $\left(\mathbf{u}_{N}^{n+\frac{i}{2}}, v_{N}^{n+\frac{i}{2}}, \eta_{N}^{n+\frac{i}{2}}\right)$.

5.2.1 Problem A1: The structure elastodynamics problem. The semi-discretization of the structure elastodynamics problem involving nonlinear Koiter membrane terms has to be performed in a different way from the semi-discretization of the corresponding linear problem, which was discretized in [36] by the Backward Euler scheme. Discretization via the Backward Euler scheme used in [36] would not yield a uniform estimate (uniform with respect to $\Delta t$ ) in the nonlinear case. To get around this difficulty we employ the following time discretization of the Gateux derivative $\mathbf{G}^{\prime}$ :

$$
\begin{aligned}
\mathbf{G}^{\prime}\left(\eta^{n+1}, \eta^{n}\right) \xi & :=\frac{1}{2}\left(\mathbf{G}^{\prime}\left(\eta^{n+1}\right)+\mathbf{G}^{\prime}\left(\eta^{n}\right)\right) \xi \\
& =\left(\begin{array}{cc}
\partial_{z}\left(\bar{\eta}^{n+1}\right) \partial_{z} \xi & \frac{1}{2}\left(\partial_{z}\left(\bar{\eta}^{n+1}\right) \partial_{\theta} \xi+\partial_{\theta}\left(\bar{\eta}^{n+1}\right) \partial_{z} \xi\right) \\
\frac{1}{2}\left(\partial_{z}\left(\bar{\eta}^{n+1}\right) \partial_{\theta} \xi+\partial_{\theta}\left(\bar{\eta}^{n+1}\right) \partial_{z} \xi\right) & \left(R+\bar{\eta}^{n+1} \xi+\partial_{\theta}\left(\bar{\eta}^{n+1}\right) \partial_{\theta} \xi\right.
\end{array}\right)
\end{aligned}
$$

where

$$
\bar{\eta}^{n+1}:=\frac{\eta^{n+1}+\eta^{n}}{2} .
$$

This approximation of the Gateux derivative is chosen so that the energy of the semi-discretized problem mimics the energy of the continuous problem. In particular, as we shall see in Proposition 5, equation (5.4) implies

$$
\mathbf{G}^{\prime}\left(\eta^{n+\frac{1}{2}}, \eta^{n}\right) \frac{\eta^{n+\frac{1}{2}}-\eta^{n}}{\Delta t}=\frac{1}{\Delta t}\left(\mathbf{G}\left(\eta^{n+\frac{1}{2}}\right)-\mathbf{G}\left(\eta^{n}\right)\right) \approx \partial_{t} \mathbf{G}(\eta)=\mathbf{G}^{\prime}(\eta) \partial_{t} \eta,
$$

which is crucial for the derivation of a discrete energy equality, which will eventually imply uniform boundedness of the approximating solution sequence.

The structure elastodynamics problem can now be written as follows. First, in this step $\mathbf{u}$ does not change, and so

$$
\mathbf{u}^{n+\frac{1}{2}}=\mathbf{u}^{n} .
$$

We define $\left(v^{n+\frac{1}{2}}, \eta^{n+\frac{1}{2}}\right) \in H_{0}^{2}(\omega) \times H_{0}^{2}(\omega)$ as a solution of the following problem written in weak form:

$$
\begin{gathered}
\int_{\omega} \frac{\eta^{n+\frac{1}{2}}-\eta^{n}}{\Delta t} \phi=\int_{\omega} v^{n+\frac{1}{2}} \phi \\
\rho_{s} h \int_{\omega} \frac{v^{n+\frac{1}{2}}-v^{n}}{\Delta t} \psi+\frac{h}{2} \int_{\omega} \mathrm{Q} \mathbf{G}\left(\eta^{n+\frac{1}{2}}\right): \mathbf{G}^{\prime}\left(\eta^{n+\frac{1}{2}}, \eta^{n}\right) \psi+\varepsilon \int_{\omega} \Delta \eta^{n+\frac{1}{2}} \Delta \psi=0,
\end{gathered}
$$

for all $(\phi, \psi) \in L^{2}(\omega) \times H_{0}^{2}(\omega)$. Notice that system (5.5) is not linear, and its nonlinearity is of the same type as the nonlinearity of the original membrane shell system (2.8). However, we can prove 
the existence of a unique weak solution to this problem, and an energy equality which will help us obtain uniform energy estimates for the full, semi-discretized problem.

We start by showing the existence of a weak solution to the nonlinear structure sub-problem (5.5), which is one of the main new ingredients of the present work. In contrast with the linear case, where the existence of a unique weak solution to the structure sub-problem was provided by the Lax-Milgram Lemma, here we use the Schaefer's Fixed Point Theorem to obtain the existence of a unique solution to the corresponding nonlinear structure sub-problem.

Proposition 3 For each fixed $\Delta t>0$, problem (5.5) has a unique solution $\left(v^{n+\frac{1}{2}}, \eta^{n+\frac{1}{2}}\right) \in$ $H_{0}^{2}(\omega) \times H_{0}^{2}(\omega)$.

Proof. We start by rewriting problem (5.5) in terms of the unknown $\eta^{n+1 / 2}$. Namely, we eliminate the unknown $v^{n+\frac{1}{2}}$ from (5.5) to obtain:

$$
\begin{array}{r}
\varrho_{K} h \int_{\omega} \eta^{n+\frac{1}{2}} \psi+(\Delta t)^{2} \frac{h}{2} \int_{\omega} Q \mathbf{G}\left(\eta^{n+\frac{1}{2}}\right) \cdot \mathbf{G}^{\prime}\left(\eta^{n+\frac{1}{2}}, \eta^{n}\right) \psi+(\Delta t)^{2} \varepsilon \int_{\omega} \Delta \eta^{n+1 / 2} \Delta \psi \\
=\varrho_{K} h\left(\int_{\omega} \eta^{n} \psi+\Delta t \int_{\omega} v^{n} \psi\right), \quad \psi \in H_{0}^{2}(\omega) .
\end{array}
$$

We will prove the existence of a (unique) solution to this problem by using the Schaefer's Fixed Point Theorem 4 below. For this purpose we introduce an operator

$$
B: W^{1,4}(\omega) \rightarrow W^{1,4}(\omega)
$$

which, to each $\zeta \in W^{1,4}(\omega)$ associates a $B(\zeta) \in H_{0}^{2}(\omega)$ such that

$$
\begin{aligned}
& \varrho_{K} h \int_{\omega} B(\zeta) \psi+(\Delta t)^{2} \varepsilon \int_{\omega} \Delta B(\zeta) \Delta \psi= \\
& \quad-(\Delta t)^{2} \frac{h}{2} \int_{\omega} \mathrm{QG}(\zeta) \cdot \mathbf{G}^{\prime}\left(\zeta, \eta^{n}\right) \psi+\varrho_{K} h\left(\int_{\omega} \eta^{n} \psi+\Delta t \int_{\omega} v^{n} \psi\right), \forall \psi \in H_{0}^{2}(\omega) .
\end{aligned}
$$

Existence of a unique solution $B(\zeta)$ satisfying (5.7) follows directly from the Lax-Milgram Lemma applied to the following bilinear form:

$$
b(\eta, \psi)=\varrho_{K} h \int_{\omega} \eta \psi+(\Delta t)^{2} \varepsilon \int_{\omega} \Delta \eta \Delta \psi, \quad \eta, \psi \in H_{0}^{2}(\omega)
$$

Furthermore, we have $B(\zeta) \in H_{0}^{2}(\omega) \subset W^{1,4}(\omega)$. Therefore, we proved that $B$ is a well defined operator.

To show that $B$ has a fixed point (which is a solution of (5.6)), we use the Schaefer's fixed point theorem (see e.g., [42] or [17] pp. 280-281):

THEOREM 4 (Schaefer's Fixed Point Theorem) Suppose $B: X \rightarrow X$ is a continuous and compact mapping from a Banach space $X$ into itself. Assume further that the set

$$
\{u \in X: u=\lambda B u, \text { for some } 0 \leqslant \lambda \leqslant 1\}
$$

is bounded. Then $B$ has a fixed point. 
Let us prove that $B$ satisfies all the assumption of Schaefer's Theorem.

- $B$ is compact because solutions of problem (5.7) are $H^{2}$ functions. Therefore, $\operatorname{Im}(B) \subset H_{0}^{2}(\omega)$. Now, compactness of operator $B$ follows from the compactness of the embedding $H^{2}(\omega) \hookrightarrow$ $W^{1,4}(\omega)$.

- To prove that $B$ satisfies (5.9), let $0 \leqslant \lambda \leqslant 1$ and $\eta=\lambda B(\eta)$. Then $\eta$ satisfies the following variational equality:

$$
\begin{aligned}
\varrho_{K} h \int_{\omega} \eta \psi+(\Delta t)^{2} \varepsilon \int_{\omega} \Delta \eta \Delta \psi+\lambda(\Delta t)^{2} \frac{h}{2} \int_{\omega} Q \mathbf{G}(\eta) \cdot \mathbf{G}^{\prime}\left(\eta, \eta^{n}\right) \psi \\
=\lambda \varrho_{K} h\left(\int_{\omega} \eta^{n} \psi+\Delta t \int_{\omega} v^{n} \psi\right), \psi \in H_{0}^{2}(\omega) .
\end{aligned}
$$

Introduce $v:=\left(\eta-\eta^{n}\right) / \Delta t$ and rewrite 5.10 in the following way:

$$
\begin{aligned}
\varrho_{K} h \Delta t \int_{\omega}\left(v-v^{n}\right) \psi+(\Delta t)^{2} \varepsilon \int_{\omega} \Delta \eta \Delta \psi+\lambda(\Delta t)^{2} \frac{h}{2} \int_{\omega} Q \mathbf{G}(\eta) \cdot \mathbf{G}^{\prime}\left(\eta, \eta^{n}\right) \psi \\
\quad=(\lambda-1) \varrho_{K} h\left(\int_{\omega} \eta^{n} \psi+\Delta t \int_{\omega} v^{n} \psi\right), \psi \in H_{0}^{2}(\omega)
\end{aligned}
$$

By taking $v$ as a test function and by analogous reasoning as in the proof of Proposition 5 we get

$$
\begin{aligned}
& \frac{\varrho_{K} h \Delta t}{2}\left(\|v\|_{L^{2}(\omega)}^{2}+\right.\left.\left\|v-v^{n}\right\|_{L^{2}(\omega)}^{2}\right) \\
&+\lambda(\Delta t)^{2} \frac{h}{4}\left(\int_{\omega} Q \mathbf{G}(\eta) \cdot \mathbf{G}(\eta)+\int_{\omega} Q\left(\mathbf{G}(\eta)-\mathbf{G}\left(\eta^{n}\right)\right) \cdot\left(\mathbf{G}(\eta)-\mathbf{G}\left(\eta^{n}\right)\right)\right) \\
&+(\Delta t)^{2} \frac{\varepsilon}{2}\left(\|\Delta \eta\|_{L^{2}(\omega)}^{2}+\left\|\Delta \eta-\Delta \eta^{n}\right\|_{L^{2}(\omega)}^{2}\right) \\
&=(\lambda-1) \varrho_{K} h\left(\int_{\omega} \eta^{n} v+\Delta t \int_{\omega} v^{n} v\right) \\
& \quad+\frac{\varrho_{K} h \Delta t}{2}\left\|v^{n}\right\|_{L^{2}(\omega)}^{2}+\lambda(\Delta t)^{2} \frac{h}{4}\left\|\eta^{n}\right\|_{\gamma}^{4}+(\Delta t)^{2} \frac{\varepsilon}{2}\left\|\Delta \eta^{n}\right\|_{L^{2}(\omega)}^{2}
\end{aligned}
$$

The first term on the right-hand side of (5.12) can be estimated as follows:

$$
\begin{aligned}
\left|(\lambda-1) \varrho_{K} h\left(\int_{\omega} \eta^{n} v+\Delta t \int_{\omega} v^{n} v\right)\right| & \leqslant(1-\lambda) \varrho_{K} h\|v\|_{L^{2}(\omega)}\left\|\eta^{n}+\Delta t v^{n}\right\|_{L^{2}(\omega)} \\
& \leqslant \frac{(1-\lambda)}{2} \varrho_{K} h\left(s\|v\|_{L^{2}(\omega)}^{2}+\frac{1}{s}\left\|\eta^{n}+\Delta t v^{n}\right\|_{L^{2}(\omega)}^{2}\right),
\end{aligned}
$$

where $s>0$. If we take $s=\Delta t / 2$ we can absorb $\|v\|_{L^{2}(\omega)}^{2}$ in the left-hand side. The other terms on the right hand side of (5.12) depend only on the given data $\Delta t, \eta^{n}, h, \varepsilon$ and $v^{n}$. Therefore they are bounded from above by some constant $C\left(\Delta t, \varepsilon, \eta^{n}, v^{n}, h\right)$. Recall that we are keeping $\Delta t$ fixed here. Now, because $Q$ is a positive operator, we have

$$
(\Delta t)^{2} \varepsilon\|\Delta \eta\|^{2} \leqslant \frac{(1-\lambda)}{2 \Delta t} \varrho_{K} h\left\|\eta^{n}+\Delta t v^{n}\right\|_{L^{2}(\omega)}^{2}+C\left(\Delta t, \varepsilon, \eta^{n}, v^{n}, h\right) .
$$


By the Sobolev embeddings, we have:

$$
\|\eta\|_{W^{1,4}(\omega)} \leqslant C\|\eta\|_{H^{2}(\omega)} \leqslant C\|\Delta \eta\|_{L^{2}(\omega)} \leqslant C\left(\Delta t, \varepsilon, \eta^{n}, v^{n}, h\right) .
$$

Therefore we have proven (5.9).

- Now, it only remains to prove that $B$ is continuous. Let $\zeta_{k} \rightarrow \zeta$ in $W^{1,4}(\omega)$. We need to prove that

$$
B\left(\zeta_{k}\right)=: \eta_{k} \rightarrow B(\zeta)=: \eta \text { in } W^{1,4}(\omega) .
$$

Let $r_{k}=\eta-\eta_{k}$. By using the definition (5.7) of operator $B$ and applying it to $\zeta_{k}$ and $\zeta$, and then subtracting one from the other, we get the following equation for $r_{k}$ :

$$
\begin{aligned}
& \varrho_{K} h \int_{\omega} r_{k} \psi+(\Delta t)^{2} \varepsilon \int_{\omega} \Delta r_{k} \Delta \psi \\
& =(\Delta t)^{2} \frac{h}{2} \int_{\omega} Q \mathbf{G}\left(\zeta_{k}\right): \mathbf{G}^{\prime}\left(\zeta_{k}, \eta^{n}\right) \psi-(\Delta t)^{2} \frac{h}{2} \int_{\omega} Q \mathbf{G}(\zeta): \mathbf{G}^{\prime}\left(\zeta, \eta^{n}\right) \psi, \psi \in H_{0}^{2}(\omega) .
\end{aligned}
$$

Recall that $\eta^{n}$ is fixed in this problem (it is a given data), and it is determined from the fact that we are working with a given, fixed $\Delta t$. In proving continuity of $B$ we are only interested in what happens as $k \rightarrow \infty$. Now, from the definition of $\mathbf{G}(\zeta)$, given by (2.4), and the convergence properties of the sequence $\zeta_{k}$, we get:

$$
\mathbf{G}\left(\zeta_{k}\right) \rightarrow \mathbf{G}(\zeta) \text { in } L^{2}(\omega)
$$

Similarly, we have:

$$
\mathbf{G}^{\prime}\left(\zeta_{k}, \eta^{n}\right) \psi \rightarrow \mathbf{G}^{\prime}\left(\zeta, \eta^{n}\right) \psi \text { in } L^{2}(\omega), \psi \in H_{0}^{2}(\omega) .
$$

By taking $r_{k}$ as a test function in (5.13) we get:

$$
\begin{aligned}
& \varrho_{K} h\left\|r_{k}\right\|_{L^{2}(\omega)}^{2}+(\Delta t)^{2} \varepsilon\left\|\Delta r_{k}\right\|_{L^{2}(\omega)}^{2} \\
&=(\Delta t)^{2} \frac{h}{2} \int_{\omega} Q \mathbf{G}\left(\zeta_{k}\right): \mathbf{G}^{\prime}\left(\zeta_{k}, \eta^{n}\right) r_{k}-(\Delta t)^{2} \frac{h}{2} \int_{\omega} Q \mathbf{G}(\zeta): \mathbf{G}^{\prime}\left(\zeta, \eta^{n}\right) r_{k} \\
&=-(\Delta t)^{2} \frac{h}{2} \int_{\omega}\left(Q \mathbf{G}(\zeta):\left(\left(\mathbf{G}^{\prime}\left(\zeta, \eta^{n}\right)-\mathbf{G}^{\prime}\left(\zeta_{k}, \eta^{n}\right)\right)\right.\right. \\
&\left.+Q\left(\mathbf{G}(\zeta)-\mathbf{G}\left(\zeta_{k}\right)\right): \mathbf{G}^{\prime}\left(\zeta_{k}, \eta^{n}\right)\right) r_{k}
\end{aligned}
$$

The convergence properties of $\mathbf{G}\left(\zeta_{k}\right)$ and $\mathbf{G}^{\prime}\left(\zeta_{k}, \eta^{n}\right)$ then imply

$$
r_{k}=\eta_{k}-\eta \rightarrow 0 \text { in } H_{0}^{2}(\omega) \text {. }
$$

The continuity of $B$ now follows directly from the Sobolev embedding $H^{2}(\omega) \hookrightarrow W^{1,4}(\omega)$.

PROPOSITION 5 For each fixed $\Delta t>0$, solution of problem (5.5) satisfies the following discrete energy equality:

$$
\begin{aligned}
E_{N}^{n+\frac{1}{2}}+\frac{1}{2}\left(\rho_{s} h\left\|v^{n+\frac{1}{2}}-v^{n}\right\|^{2}+\frac{h}{2} \int_{\omega} \mathbb{Q}\left(\mathbf{G}\left(\eta^{n+\frac{1}{2}}\right)\right.\right. & \left.-\mathbf{G}\left(\eta^{n}\right)\right):\left(\mathbf{G}\left(\eta^{n+\frac{1}{2}}\right)-\mathbf{G}\left(\eta^{n}\right)\right) \\
& \left.+\varepsilon\left\|\Delta\left(\eta^{n+\frac{1}{2}}-\eta^{n}\right)\right\|_{L^{2}(\omega)}^{2}\right)=E_{N}^{n},
\end{aligned}
$$

where the kinetic energy $E_{N}^{n}$ is defined in (5.2). 
Proof. From Proposition 3 we have $v^{n+\frac{1}{2}} \in H_{0}^{2}(\omega)$. We can therefore take $\Delta t v^{n+\frac{1}{2}}=\eta^{n+\frac{1}{2}}-\eta^{n}$ as a test function in the second equation of (5.5). Now, we use the following identity, which can be obtained by a straightforward calculation from (5.4) and (2.4):

$$
\mathbf{G}^{\prime}\left(\eta^{n+\frac{1}{2}}, \eta^{n}\right)\left(\eta^{n+\frac{1}{2}}-\eta^{n}\right)=\mathbf{G}\left(\eta^{n+\frac{1}{2}}\right)-\mathbf{G}\left(\eta^{n}\right)
$$

We would like to emphasize that this identity was the reason why we introduced the specific discretization of $\mathbf{G}^{\prime}\left(\eta^{n+\frac{1}{2}}, \eta^{n}\right)$, which is dictated by the type of nonlinearity in $\mathbf{G}(\eta)$. Namely, this discretization mimics the continuous problem well in a following sense:

$$
\mathbf{G}^{\prime}\left(\eta^{n+\frac{1}{2}}, \eta^{n}\right) \frac{\eta^{n+\frac{1}{2}}-\eta^{n}}{\Delta t}=\frac{1}{\Delta t}\left(\mathbf{G}\left(\eta^{n+\frac{1}{2}}\right)-\mathbf{G}\left(\eta^{n}\right)\right) \approx \partial_{t} \mathbf{G}(\eta)=\mathbf{G}^{\prime}(\eta) \partial_{t} \eta .
$$

Now, we can finish the proof in a standard way, as in [36], by using the algebraic identity $(a-b) \cdot a=$ $\frac{1}{2}\left(|a|^{2}+|a-b|^{2}-|b|^{2}\right)$ and the symmetry property of the elasticity tensor $Q$ defined by (2.2).

In the next section we study the fluid sub-problem. Due to the modularity of our approach, the fluid sub-problem is conveniently the same as the fluid sub-problem in the FSI problem involving the linearly elastic cylindrical Koiter model studied in [38]. Here is where the robustness of our approach becomes useful. We summarize the weak formulation and state the results regarding the existence and energy estimate, which are the same as those presented in [36, 38].

5.2.2 Problem A2: The fluid problem. In this step $\eta$ does not change, and so

$$
\eta^{n+1}=\eta^{n+\frac{1}{2}}
$$

Define $\left(\mathbf{u}^{n+1}, v^{n+1}\right) \in V_{F}^{\eta^{n}} \times L^{2}(\omega)$ by requiring that for all $(\mathbf{q}, \xi) \in \mathcal{V}_{F}^{\eta^{n}} \times L^{2}(\omega)$ such that $\mathbf{q}_{\mid \Gamma}=\xi \mathbf{e}_{r}$, the following weak formulation holds:

$$
\begin{gathered}
\rho_{f} \int_{\Omega}\left(R+\eta^{n}\right)^{2}\left(\frac{\mathbf{u}^{n+1}-\mathbf{u}^{n+\frac{1}{2}}}{\Delta t} \cdot \mathbf{q}+\frac{1}{2}\left[\left(\mathbf{u}^{n}-\mathbf{w}^{n+\frac{1}{2}}\right) \cdot \nabla^{\eta^{n}}\right] \mathbf{u}^{n+1} \cdot \mathbf{q}\right. \\
\left.-\frac{1}{2}\left[\left(\mathbf{u}^{n}-\mathbf{w}^{n+\frac{1}{2}}\right) \cdot \nabla^{\eta^{n}}\right] \mathbf{q} \cdot \mathbf{u}^{n+1}\right)+\rho_{f} \int_{\Omega}\left(R+\frac{\eta^{n}+\eta^{n+1}}{2}\right) v^{n+\frac{1}{2}} \mathbf{u}^{n+1} \cdot \mathbf{q} \\
\quad+2 \mu_{F} \int_{\Omega}\left(R+\eta^{n}\right)^{2} \mathbf{D}^{\eta^{n}}(\mathbf{u}): \mathbf{D}^{\eta^{n}}(\mathbf{q})+R \rho_{K} h \int_{\omega} \frac{v^{n+1}-v^{n+\frac{1}{2}}}{\Delta t} \xi \\
=R\left(P_{\text {in }}^{n} \int_{\Gamma_{\text {in }}}\left(q_{z}\right)_{\mid z=0}-P_{\text {out }}^{n} \int_{\Gamma_{\text {out }}}\left(q_{z}\right)_{\mid z=L}\right), \\
\quad \text { with } \nabla^{\eta^{n}} \cdot \mathbf{u}^{n+1}=0, \quad \mathbf{u}_{\mid \Gamma}^{n+1}=v^{n+1} \mathbf{e}_{r},
\end{gathered}
$$

where $P_{\text {in } / \text { out }}^{n}=\frac{1}{\Delta t} \int_{n \Delta t}^{(n+1) \Delta t} P_{\text {in } / \text { out }}(t) d t$ and $\mathbf{w}^{n+\frac{1}{2}}=v^{n+\frac{1}{2}}\left(\begin{array}{l}0 \\ x \\ y\end{array}\right)$.

Proposition 6 ([38], Proposition 4.3) Let $\Delta t>0$, and assume that $\eta^{n}$ are such that $R+\eta^{n} \geqslant$ $R_{\min }>0, n=0, \ldots, N$. Then, the fluid sub-problem defined by (5.17) has a unique weak solution $\left(\mathbf{u}^{n+1}, v^{n+1}\right) \in V_{F}^{\eta^{n}} \times H_{0}^{2}(\omega)$. 
Proposition 7 ([38], Proposition 4.4) For each fixed $\Delta t>0$, solution of problem (5.17) satisfies the following discrete energy inequality:

$$
\begin{aligned}
& E_{N}^{n+1}+\frac{\rho_{f}}{2} \int_{\Omega}\left(R+\eta^{n}\right)\left|\mathbf{u}^{n+1}-\mathbf{u}^{n}\right|^{2}+\frac{\rho_{s} h}{2}\left\|v^{n+1}-v^{n+\frac{1}{2}}\right\|_{L^{2}(\omega)}^{2}+D_{N}^{n+1} \\
& \leqslant E_{N}^{n+\frac{1}{2}}+C \Delta t\left(\left(P_{\text {in }}^{n}\right)^{2}+\left(P_{\text {out }}^{n}\right)^{2}\right)
\end{aligned}
$$

where the kinetic energy $E_{N}^{n}$ and dissipation $D_{N}^{n}$ are defined in (5.2) and (5.3), and the constant $C$ depends only on the parameters in the problem, and not on $\Delta t$ (or $N)$.

We pause for a second, and summarize what we have accomplished so far. For a given $\Delta t>0$ we divided the time interval $(0, T)$ into $N=T / \Delta t$ sub-intervals $\left(t^{n}, t^{n+1}\right), n=0, \ldots, N-1$. On each sub-interval $\left(t^{n}, t^{n+1}\right)$ we "solved" the coupled FSI problem by applying the Lie splitting scheme. First we solved for the structure position (Problem A1) and then for the fluid flow (Problem A2). We have just shown that each sub-problem has a unique solution, provided that $R+\eta^{n} \geqslant R_{\min }>0, n=$ $0, \ldots, N$, and that its solution satisfies an energy estimate. When combined, the two energy estimates provide a discrete version of the energy estimate (3.2). Thus, for each $\Delta t$ we have a time-marching, splitting scheme which defines an approximate solution on $(0, T)$ of our main FSI problem defined in Problem 2, and is such that for each $\Delta t$ the approximate FSI solution satisfies a discrete version of the energy estimate for the continuous problem.

What we would like to ultimately show is that, as $\Delta t \rightarrow 0$, the sequence of solutions parameterized by $N$ (or $\Delta t$ ), converges to a weak solution of Problem 2. Furthermore, we also need to show that $R+\eta^{n} \geqslant R_{\min }>0$ is satisfied for each $n=0, \ldots, N-1$. In order to obtain this result, it is crucial to show that the discrete energy of the approximate FSI solutions defined for each $\Delta t$, is uniformly bounded, independently of $\Delta t$ (or $N$ ). This result is obtained by the following Lemma.

LEMMA 8 (The uniform energy estimates) Let $\Delta t>0$ and $N=T / \Delta t>0$. Furthermore, let $E_{N}^{n+\frac{1}{2}}, E_{N}^{n+1}$, and $D_{N}^{j}$ be the kinetic energy and dissipation given by (5.2) and (5.3), respectively.

There exists a constant $C>0$ independent of $\Delta t$ (and $N$ ), which depends only on the parameters in the problem, on the kinetic energy of the initial data $E_{0}$, and on the energy norm of the inlet and outlet data $\left\|P_{\text {in/out }}\right\|_{L^{2}(0, T)}^{2}$, such that the following estimates hold:

1. $E_{N}^{n+\frac{1}{2}} \leqslant C, E_{N}^{n+1} \leqslant C$, for all $n=0, \ldots, N-1$,

2. $\sum_{j=1}^{N} D_{N}^{j} \leqslant C$,

3. $\sum_{n=0}^{N-1}\left(\int_{\Omega}\left(R+\eta^{n}\right)^{2}\left|\mathbf{u}^{n+1}-\mathbf{u}^{n}\right|^{2}+\left\|v^{n+1}-v^{n+\frac{1}{2}}\right\|_{L^{2}(\omega)}^{2}+\left\|v^{n+\frac{1}{2}}-v^{n}\right\|_{L^{2}(\omega)}^{2}\right) \leqslant C$,

4. $\sum_{n=0}^{N-1}\left(\int_{\omega} Q\left(\mathbf{G}\left(\eta^{n+1}\right)-\mathbf{G}\left(\eta^{n}\right)\right):\left(\mathbf{G}\left(\eta^{n+1}\right)-\mathbf{G}\left(\eta^{n}\right)\right)+\varepsilon\left\|\Delta\left(\eta^{n+1}-\eta^{n}\right)\right\|_{L^{2}(\omega)}^{2} B i g\right) \leqslant C$. In fact, $C=E_{0}+\tilde{C}\left(\left\|P_{i n}\right\|_{L^{2}(0, T)}^{2}+\left\|P_{o u t}\right\|_{L^{2}(0, T)}^{2}\right)$, where $\tilde{C}$ is the constant from (5.18), which depends only on the parameters in the problem.

Proof. The proof follows directly from the estimates (5.15) and (5.18) in the same way as in the proof of Lemma 1 in [36]. Note that the $\Delta t$ appearing on the right hand-side of (5.18) is absorbed in the definition of the $L^{2}$-norms of $P_{\text {in } / \text { out }}$ over the time interval $(0, T),\left\|P_{\text {in } / \text { out }}\right\|_{L^{2}(0, T)}^{2}$. 


\section{Convergence of approximate solutions}

In the previous sections, for each given $\Delta t=T / N$ we constructed approximate sequences $\left(\mathbf{u}_{N}^{n+\frac{i}{2}}, v_{N}^{n-\frac{i}{2}}, \eta_{N}^{n+\frac{i}{2}}\right), i=0,1, n=1, \ldots, N, N \in \mathbb{N}$, which are defined at discrete points $t_{0}, t_{1}, \ldots, t_{N}$, and proved that the approximating sequences satisfy the uniform energy estimates in Lemma 8.

Now we define approximate solutions on $(0, T)$ of Problem 2 to be the functions which are piece-wise constant on each sub-interval $((n-1) \Delta t, n \Delta t], n=1, \ldots, N$ of $(0, T)$, such that for $t \in((n-1) \Delta t, n \Delta t], n=1 \ldots N$,

$$
\mathbf{u}_{N}(t, .)=\mathbf{u}_{N}^{n}, \eta_{N}(t, .)=\eta_{N}^{n}, \quad v_{N}(t, .)=v_{N}^{n}, v_{N}^{*}(t, .)=v_{N}^{n-\frac{1}{2}} .
$$

Notice that the functions $v_{N}^{*}$ are defined by the elastodynamics problem (Problem A1) to be equal to the normal component of the structure velocity (the time-derivative of the normal component of displacement of the fluid-structure interface), with the initial data at every time step given by the trace of the fluid velocity at the fluid-struture interface. The functions $v_{N}$, on the other hand, are defined by the fluid problem (Problem A2) to be the normal trace of the fluid velocity at the fluidstructure interface, obtained at every time step with the initial data which is given by the structure velocity from the previous time step. These two functions, $v_{N}^{*}$ and $v_{N}$, are not necessarily the same. They are a result of the fact that the kinematic coupling condition is satisfied in our splitting scheme asynchronously. This is an interesting feature of the scheme which is useful in, for example, the implementation of this scheme for numerical simulation of FSI problems in which contact between structures needs to be resolved (e.g., closure of heart valves). Because of this particular property, the proposed scheme is particularly suitable for solving this class of FSI problems since it would allow detachment (opening of the valve leaflets) in a natural way once two structures have been in contact (closed valve leaflets). This is, for example, not the case with monolithic schemes. We will show, however, that in the limit, as $\Delta t \rightarrow 0$, the two sequences converge to the same value, which corresponds to the kinematic coupling condition being satisfies by the limiting solution as $N \rightarrow \infty$.

In the current paper, however, we prove "global existence" of a weak solution as long as the structures do not touch each other. This was a necessary condition in Proposition 6 under which the existence of a weak solution to Problem A2 can be proved. We now show that this condition is satisfied for a non zero time interval $(0, T), T>0$, provided that the initial data satisfy the same property (that the cross-section of the initial cylinder is strictly greater than zero). Later in the paper we will show that this time $T>0$ is not small, namely, that our existence result is not local in time.

Before we present the result, an important remark is in order.

Remark. Thanks to the modularity and robustness of our approach, we can proceed in this paper in a similar way as in [38] where the existence of a weak solution was proved for an FSI problem between an incompressible, viscous Newtonian fluid and a linearly elastic cylindrical Koiter shell in 3D. Namely, since the fluid sub-problem is the same as in [38], the estimates and convergence results for the fluid velocity stay the same. The structure problem, although different due to the nonlinearity in the membrane terms, retains the same convergence properties as in the linear case. Namely, because of the nonlinearity in the nonlinear membrane terms, Lemma 8 implies uniform estimates in $L^{\infty}\left(0, T ; W^{1,4}(\omega)\right)$, which provide higher regularity than the uniform estimates in the linear case, presented in Lemma 4.1 in [38] implying uniform bounds only in $L^{\infty}\left(0, T ; L^{2}(\omega)\right)$. However, in both cases the dominant terms associated with bending rigidity of Koiter shells are linear, and they 
provide uniform bounds of the displacement in $L^{\infty}\left(0, T ; H^{2}(\omega)\right)$. Due to the Sobolev embedding $H^{2}(\omega) \hookrightarrow W^{1,4}(\omega)$, the highest-order, shell terms are the ones that determine the final convergence result, which is analogous to the result obtained in [38]. However, in the present paper we will still have to deal with the lower-order nonlinear terms. In what follows, we state the main results, show the parts of the proofs that involve new calculations involving nonlinear terms, and refer the reader to Section 5 in [38] for further details and proofs that are anologous to the linear case (see also Sections 6 and 8 in [36]).

Using the uniform estimates provided by Lemma 8, an interpolation inequality for Sobolev spaces and a Sobolev embedding result in the same way as in the proof of Proposition 5.1 in [38], one can prove the following result:

Proposition 9 ([38], Proposition 5.1) Sequence $\left(\eta_{N}\right)_{N \in \mathbb{N}}$ is uniformly bounded in

$$
L^{\infty}\left(0, T ; H_{0}^{2}(\omega)\right) \text {. }
$$

Moreover, for $T$ small enough, we have

$$
0<R_{\min } \leqslant R+\eta_{N}(t, z, \theta) \leqslant R_{\max }, \quad \forall N \in \mathbb{N},(z, \theta) \in \omega, t \in(0, T) .
$$

This proposition shows that there exists a time interval $(0, T)$ where all the approximate solutions defined at the beginning of this section are well defined, and that the approximate displacement is uniformly bounded in $L^{\infty}\left(0, T ; H_{0}^{2}(\omega)\right)$ on that interval. If we could have uniform boundedness of all the approximate sequences and their appropriate derivatives, then we could obtain weak (or weak*) convergence of the corresponding subsequences, which is a first step toward proving convergence of the approximations defined with our splitting scheme to a weak solution.

The main ingredient in obtaining uniform boundedness and weak/weak* convergence of approximate subsequences is Lemma 8. This lemma implies uniform boundedness of all the approximate sequences, except for the gradient of the fluid velocity. Lemma 8 does imply uniform boundedness of the transformed symmetrized gradient of the velocity, defined on the domain which is determined by the displacement $\eta_{N}$ from the previous time step, i.e., at $t-\Delta t$. To denote this time-shift by $\Delta t$, or in general by some $h$, we introduce the time-shift function denoted by $\tau_{h}$ as the translation of a given function $f$ in time by $h$ :

$$
\tau_{h} f(t, .)=f(t-h, .), \quad h \in \mathbb{R} .
$$

It can be easily shown that Lemma 8 implies the following uniform boundedness results [38]:

PROPOSITION 10 The following statements hold:

1. $\left(v_{N}\right)_{n \in \mathbb{N}}$ is uniformly bounded in $L^{\infty}\left(0, T ; L^{2}(\omega)\right)$.

2. $\left(v_{N}^{*}\right)_{n \in \mathbb{N}}$ is uniformly bounded in $L^{\infty}\left(0, T ; L^{2}(\omega)\right)$.

3. $\left(\mathbf{u}_{N}\right)_{n \in \mathbb{N}}$ is uniformly bounded in $L^{\infty}\left(0, T ; L^{2}(\Omega)\right)$.

4. $\left(\mathbf{D}^{\tau_{\Delta t} \eta_{N}}\left(\mathbf{u}_{N}\right)\right)_{n \in \mathbb{N}}$ is uniformly bounded in $L^{2}((0, T) \times \Omega)$.

To show convergence of approximate solutions to a weak solution of the FSI problem, we need to have information about the behavior of the transformed gradient of the fluid velocity $\nabla^{\tau_{\Delta t}} \eta_{N} \mathbf{u}_{N}$. To obtain uniform boundedness of the transformed gradient of the fluid velocity $\nabla^{\tau} \Delta t \eta_{N} \mathbf{u}_{N}$, we proceed in the same way as in [36, 38] and use Korn's inequality. Now, since we are working with the transformed gradients via the ALE mappings onto a fixed domain, the Korn's constant in 
general will depend on the fluid domain. This is a difficulty that needs to be overcome any time a moving-boundary problem is solved by mapping a sequence of approximating problems onto a fixed domain. It was shown in $[36,38]$ that for our particular problem in 3D there exists a universal Korn's constant, independent of the family of domains under consideration, which provides the desired uniform bound for the transformed gradient of the fluid velocity. As a consequence, the resulting gradient is uniformly bounded in $L^{2}((0, T) \times \Omega)$, and the following weak and weak* convergence results follow:

LEMMA 11 ([38], Lemma 5.1) (Weak and weak* convergence results) There exist subsequences $\left(\eta_{N}\right)_{N \in \mathbb{N}},\left(v_{N}\right)_{N \in \mathbb{N}},\left(v_{N}^{*}\right)_{N \in \mathbb{N}}$, and $\left(\mathbf{u}_{N}\right)_{N \in \mathbb{N}}$, and the functions $\eta \in L^{\infty}\left(0, T ; H_{0}^{2}(\omega)\right), v, v^{*} \in$ $L^{\infty}\left(0, T ; L^{2}(\omega)\right)$, and $\mathbf{u} \in L^{\infty}\left(0, T ; L^{2}(\Omega)\right)$, such that

$$
\begin{aligned}
\eta_{N} & \rightarrow \eta \text { weakly* in } L^{\infty}\left(0, T ; H_{0}^{2}(\omega)\right), \\
v_{N} & \rightarrow v \text { weakly* in } L^{\infty}\left(0, T ; L^{2}(\omega)\right), \\
v_{N}^{*} & \rightarrow v^{*} \text { weakly* in } L^{\infty}\left(0, T ; L^{2}(\omega)\right), \\
\mathbf{u}_{N} & \rightarrow \mathbf{u} \text { weakly* in } L^{\infty}\left(0, T ; L^{2}(\Omega)\right), \\
\nabla^{\tau_{\Delta t} \eta_{N}} \mathbf{u}_{N} & \rightarrow \mathbf{M} \text { weakly in } L^{2}((0, T) \times \Omega) .
\end{aligned}
$$

Furthermore,

$$
v=v^{*} \text {. }
$$

Notice that at this point we still cannot prove that the transformed gradients $\nabla^{\tau} \Delta t \eta_{N} \mathbf{u}_{N}$ of the approximate fluid velocities converge to the transformed gradient $\nabla^{\eta} \mathbf{u}$ of the limit function. We also do not know yet if that limit velocity $\mathbf{u}$ is in $L^{2}\left(0, T ; H^{1}(\Omega)\right)$. This is a consequence of the fact that $\eta$ is not necessarily a Lipschitz function and therefore the ALE mapping $A_{\eta}$ is not regular enough to preserve the regularity of the solution defined on the deformed physical domain.

To deal with this issue and to obtain strong convergence results that will allow us to pass to the limit in the nonlinear terms to show that the limiting function is a weak solution of the FSI problem, we need to obtain a compactness result that will allow us to complete the existence proof. Our compactness result is based on Simon's theorem [41], which characterizes compact sets in $L^{p}(0, T ; X)$, where $X$ is a Banach space. See also [36] where details of the use of Simon's theorem in the proof of compactness in the $2 \mathrm{D}$ radially symmetric case are presented.

THEOREM 12 ([38], Theorem 5.1) (Main compactness result for velocities) Sequences $\left(v_{N}\right)_{N \in \mathbb{N}}$, $\left(\mathbf{u}_{N}\right)_{N \in \mathbb{N}}$ are relatively compact in $L^{2}\left(0, T ; L^{2}(\omega)\right)$ and $L^{2}\left(0, T ; L^{2}(\Omega)\right)$ respectively.

Proof. Here we just comment the main idea of the proof, and refer the reader to [36, 38] for details. As mentioned above, the proof is based on Simon's theorem, which states that for a set $F, F \hookrightarrow$ $L^{p}(0, T ; X)$, with $1 \leqslant p<\infty$ to be relatively compact in $L^{p}(0, T ; X)$, it is necessary and sufficient that the following two properties are satisfied:

(i) $\left\|\tau_{h} f-f\right\|_{L^{p}(h, T ; X)} \rightarrow 0$ as $h$ goes to zero, uniformly in $f \in F$ (integral "equicontinuity" in time), and

(ii) $\left\{\int_{t_{1}}^{t_{2}} f(t) d t: f \in F\right\}$ is relatively compact in $X, 0<t_{1}<t_{2}<T$ (spatial compactness).

The essential ingredients for proving these two properties are the uniform energy bounds given in Lemma 8. To show the integral equicontinuity in time we multiply the third inequality of Lemma 
8 by $\Delta t$ to get that the "half-order derivative in time" is uniformly bounded (i.e., constant $C$ below is independent of $\Delta t$ ):

$$
\left\|\tau_{\Delta t} \mathbf{u}_{N}-\mathbf{u}_{N}\right\|_{L^{2}((0, T) \times \Omega)}^{2}+\left\|\tau_{\Delta t} v_{N}-v_{N}\right\|_{L^{2}((0, T) \times \omega)}^{2} \leqslant C \Delta t .
$$

This is "almost" the integral equicontinuity stated in (i) above, except that in this estimate the smallness of the expression in (6.6) is estimated from above by $C \Delta t$ which is not independent of $N$ (i.e., of $\Delta t$ ). For the integral equicontinuity (i) above, the functions $(\mathbf{u})_{N \in \mathbb{N}} \in F$ and $(v)_{N \in \mathbb{N}} \in F$ need to satisfy the condition in (i) uniformly in $N \in \mathbb{N}$ (i.e., uniformly in $\Delta t$ ). This can be proved by a closer investigation of the structure of the sequences $(\mathbf{u})_{N \in \mathbb{N}} \in F$ and $(v)_{N \in \mathbb{N}} \in F$, which was done in [36], Theorem 2 (see also [37]), and so we omit the details here.

The spatial compactness for the fluid velocity is obtained as a consequence of the compactness of Sobolev embeddings and from spatial regularity of the fluid velocity (Lemma 8, statement 2) as in [38]. The spatial compactness of the structure velocity is obtained from the regularity of the fluid velocity by taking into account the kinematic coupling condition $\left(\mathbf{u}_{N}\right)_{\Gamma} \cdot \mathbf{e}_{r}=v_{N}$, and by using the trace theorem. However, there is a technical difficulty associated with this approach, which is related to the fact that the fluid-structure interface is not necessarily Lipschitz, and the sequence $\eta_{N}$ is not uniformly bounded in $W^{1, \infty}(\omega)$. This can be resolved by using results from [35] about the traces on domains which are not Lipschitz, but are sub-graphs of Hölder continuous functions. Details of the proof can be found in [38], Theorem 5.1.

To obtain compactness of $\left(\eta_{N}\right)_{N \in \mathbb{N}}$, and to be able to pass to the limit and obtain the final existence result, we need to introduce a slightly different set of approximating functions of $\mathbf{u}, v$, and $\eta$. Namely, instead of extending the values of the unknown functions at semi-discretized points $n \Delta t$ to the entire time interval of width $\Delta t$ by a constant, as was done earlier in this section, we now extend these approximate solution values to the time interval of width $\Delta t$ as a linear function of $t$. More precisely, for each fixed $\Delta t$ (or $N \in \mathbb{N}$ ), define $\tilde{\mathbf{u}}_{N}, \tilde{\eta}_{N}$ and $\tilde{v}_{N}$ to be continuous, linear on each sub-interval $[(n-1) \Delta t, n \Delta t]$, and such that

$$
\tilde{\mathbf{u}}_{N}(n \Delta t, .)=\mathbf{u}_{N}(n \Delta t, .), \tilde{v}_{N}(n \Delta t, .)=v_{N}(n \Delta t, .), \tilde{\eta}_{N}(n \Delta t, .)=\eta_{N}(n \Delta t, .),
$$

where $n=0, \ldots, N$. As we shall see, both sequences of approximating functions converge to the same limit, and they both appear in the weak formulation of the semi-discretized FSI problem (see (7.1) below). Therefore, we need results related to both approximating sequences.

First, we observe that

$$
\partial_{t} \tilde{\eta}_{N}(t)=\frac{\eta^{n+1}-\eta^{n}}{\Delta t}=\frac{\eta^{n+1 / 2}-\eta^{n}}{\Delta t}=v^{n+\frac{1}{2}}, t \in(n \Delta t,(n+1) \Delta t),
$$

which implies

$$
\partial_{t} \tilde{\eta}_{N}=v_{N}^{*} \text { a.e. on }(0, T) .
$$

By using Lemma 8, the standard interpolation inequalities, and the Arzelà-Ascoli Theorem in the same way as in [36], one obtains the following boundedness and strong convergence results for $\left(\tilde{\eta}_{N}\right)_{N \in \mathbb{N}}$ :

P1. $\left(\tilde{\eta}_{N}\right)_{N \in \mathbb{N}}$ is uniformly bounded in $C^{0,1-\alpha}\left([0, T] ; H^{2 \alpha}(0, L)\right), 0<\alpha<1$,

P2. $\tilde{\eta}_{N} \rightarrow \eta$ in $C\left([0, T] ; H^{s}(\omega)\right), 0<s<2$.

Together with the continuity in time of $\eta$ this result implies (see [36]):

$$
\eta_{N} \rightarrow \eta \text { in } L^{\infty}\left(0, T ; H^{s}(\omega)\right), \quad 0<s<2 .
$$


We can actually show even more. Namely, we will need the following convergence result for the shifted displacements $\tau_{\Delta t} \eta_{N}$ :

$$
\tau_{\Delta t} \eta_{N} \rightarrow \eta \text { in } L^{\infty}\left(0, T ; H_{0}^{s}(\omega)\right), s<2 .
$$

Namely, from P1 above we have

$$
\left\|\tilde{\eta}_{N}((n-1) \Delta t)-\tilde{\eta}_{N}(n \Delta t)\right\|_{H^{2 \alpha}(\omega)} \leqslant C|\Delta t|^{1-\alpha} .
$$

Therefore by using P2 it is immediate that

$$
\tau_{\Delta t} \tilde{\eta}_{N} \rightarrow \eta \text { in } C\left([0, T] ; H^{2 \alpha}(\omega)\right), 0<\alpha<1 .
$$

Now, from the fact that the sequences $\left(\tau_{\Delta t} \eta_{N}\right)_{N \in \mathbb{N}}$ and $\left(\tau_{\Delta t} \tilde{\eta}_{N}\right)_{N \in \mathbb{N}}$ have the same limit, we get the strong convergence result for $\left(\tau_{\Delta t} \eta_{N}\right)_{N \in \mathbb{N}}$.

Regarding the convergence results for the fluid and structure velocities of the new approximate sequences $\left(\tilde{\mathbf{u}}_{N}\right)_{N}$ and $\left(\tilde{v}_{N}\right)_{N}$ we have the following results:

$$
\begin{aligned}
& \tilde{\mathbf{u}}_{N} \rightarrow \mathbf{u} \text { in } L^{2}\left(0, T ; L^{2}(\Omega)\right), \\
& \tilde{v}_{N} \rightarrow v \text { in } L^{2}\left(0, T ; L^{2}(\omega)\right) .
\end{aligned}
$$

This follows directly from the following inequalities (see [43, p. 328])

$$
\begin{aligned}
\left\|v_{N}-\tilde{v}_{N}\right\|_{L^{2}\left(0, T ; L^{2}(\omega)\right)}^{2} & \leqslant \frac{\Delta t}{3} \sum_{n=1}^{N}\left\|v^{n+1}-v^{n}\right\|_{L^{2}(\omega)}^{2}, \\
\left\|\mathbf{u}_{N}-\tilde{\mathbf{u}}_{N}\right\|_{L^{2}\left(0, T ; L^{2}(\Omega)\right)}^{2} & \leqslant \frac{\Delta t}{3} \sum_{n=1}^{N}\left\|\mathbf{u}^{n+1}-\mathbf{u}^{n}\right\|_{L^{2}(\Omega)}^{2},
\end{aligned}
$$

and Lemma 8 which provides uniform boundedness of the sums on the right hand-sides of the inequalities.

In summary, the following strong convergence results hold for the approximating sequences $\left(\eta_{N}\right)_{N \in \mathbb{N}},\left(v_{N}\right)_{N \in \mathbb{N}},(\mathbf{u})_{n \in \mathbb{N}},\left(\tilde{\mathbf{u}}_{N}\right)_{N \in \mathbb{N}}$ and $\left(\tilde{v}_{N}\right)_{N \in \mathbb{N}}$ :

THEOREM 13 ([38], Theorem 5.2) There exist subsequences $\left(\eta_{N}\right)_{N \in \mathbb{N}},\left(v_{N}\right)_{N \in \mathbb{N}},(\mathbf{u})_{n \in \mathbb{N}}$, $\left(\tilde{\mathbf{u}}_{N}\right)_{N \in \mathbb{N}}$ and $\left(\tilde{v}_{N}\right)_{N \in \mathbb{N}}$ such that

$$
\begin{aligned}
v_{N} & \rightarrow v \text { in } L^{2}\left(0, T ; L^{2}(\omega)\right), \\
\tau_{\Delta t} \mathbf{u}_{N} & \rightarrow u \text { in } L^{2}\left(0, T ; L^{2}(\Omega)\right), \\
\tau_{\Delta t} v_{N} & \rightarrow v \text { in } L^{2}\left(0, T ; L^{2}(\omega)\right), \\
\eta_{N} & \rightarrow \eta \text { in } L^{\infty}\left(0, T ; H_{0}^{s}(\omega)\right), s<2 \\
\tau_{\Delta t} \eta_{N} & \rightarrow \eta \text { in } L^{\infty}\left(0, T ; H_{0}^{s}(\omega)\right), s<2 \\
\tilde{\mathbf{u}}_{N} & \rightarrow \mathbf{u} \text { in } L^{2}\left(0, T ; L^{2}(\Omega)\right), \\
\tilde{v}_{N} & \rightarrow v \text { in } L^{2}\left(0, T ; L^{2}(\omega)\right) .
\end{aligned}
$$




\section{The limiting problem}

We would like to show that the limiting functions satisfy the weak form (4.19) of Problem 1 as $N \rightarrow$ $\infty$. This would be relatively straight-forward if the fluid domain was not changing at every time step. To deal with the motion of the fluid domain we mapped the sequence of approximating fluid domains onto a fixed domain $\Omega$ by using a sequence of ALE mappings so that all the approximating problems are defined on the fixed domain. Unfortunately, as a result, the velocity test functions in the weak formulation of the fluid sub-problem (5.17) depend of $N$ ! More precisely, they depend on $\eta_{N}^{n}$ because of the requirement that the transformed divergence-free condition $\nabla^{\eta_{N}^{n}} \cdot \mathbf{q}=0$ must be satisfied. Passing to the limit in the weak formulation of the fluid sub-problem (5.17) when both the test functions and the unknown functions depend on $N$ is tricky, and special care needs to be taken to deal with this issue.

Our strategy is to restrict ourselves to a dense subset, call it $\chi^{\eta}(0, T)$, of the space of all test functions $Q^{\eta}(0, T)$, which will be independent of $\eta_{N}$ even for the approximating problems. In the case of Cartesian coordinates such a dense subset was constructed in [36], Section 7 (see also $[6,37,38])$. In cylindrical coordinates there is an additional technical diffculty coming from the fact that extensions of the functions defined on the interface by a constant is not divergence free as in the case of Cartesian coordinates. However, one can use the lifting operator constructed in [31] (Propostion 2.19) instead of the constant extension and analogously, as in [36], Section 7, construct a subset $\mathcal{X}^{\eta}(0, T)$ with the following properties:

- $X^{\eta}(0, T)$ is dense in $\mathbb{Q}^{\eta}(0, T)$,

- For every $(\mathbf{q}, \psi) \in \chi^{\eta}(0, T)$ there exists a $N_{q} \in \mathbb{N}$ and a sequence $\left(\mathbf{q}_{N}\right)_{N \geqslant N_{q}}$ such that $\mathbf{q}_{N} \in$ $w_{F}^{\tau_{\Delta t} \eta}(0, T)$, and

1. $\mathbf{q}_{N} \rightarrow \mathbf{q}$ uniformly on $[0, T] \times \Omega$;

2. $\nabla^{\tau \Delta t} \eta_{N}\left(\mathbf{q}_{N}\right) \rightarrow \nabla^{\eta}(\mathbf{q})$ in $L^{2}((0, T) \times \omega)$.

We are now almost ready to pass to the limit in the weak formulation of the fluid and structure sub-problems. The only thing left to show is to identify the weak limit, denoted by $\mathbf{M}$ in Lemma 11, of the transformed gradients of the fluid velocities $\nabla^{\tau} \Delta t \eta_{N} \mathbf{u}_{N}$, so that we can pass to the limit in the gradient term of the fluid sub-problem. We have been postponing this result until now because, to prove it, one needs the information about the test functions, presented above, and the information about the convergence of the sequence $\tau_{\Delta t} \eta_{N}$, given by Theorem 13. The proof is quite technical, and we refer the reader to [38, Proposition 6.1] and to [37, Proposition 7.6] for details. The result is the following:

Proposition 14 ([38, Proposition 6.1]) $\mathbf{M}=\nabla^{\eta} \mathbf{u}$, where $\mathbf{M}$, $\mathbf{u}$ and $\eta$ are the weak and weak* limits given by Lemma 11. More precisely,

$$
\nabla^{\tau} \Delta t \eta_{N} \mathbf{u}_{N} \rightarrow \nabla^{\eta} \mathbf{u} \text { weakly in } L^{2}((0, T) \times \Omega) .
$$

Now we are ready to pass to the limit. We first write the weak formulation of the coupled problem by taking $\mathbf{q}_{N}$, discussed above, for the velocity test functions. More precisely, first consider the weak formulation of the fluid sub-problem (5.17) and take $\left(\mathbf{q}_{N}(t), \psi(t)\right)$ for the test functions in (5.17). Here, $\mathbf{q}_{N}$ is a sequence of test function corresponding to $(\mathbf{q}, \psi) \in \mathcal{X}^{\eta}$. Integrate with respect to $t$ from $n \Delta t$ to $(n+1) \Delta t$. Then, consider the weak formulation for the structure sub-problem (5.5) with $\psi(t)$ as the test functions, and again integrate over the same time interval. Add the two equations together, and take the sum from $n=0, \ldots, N-1$ to get the time integrals over $(0, T)$ as follows: 


$$
\begin{gathered}
\rho_{f} \int_{0}^{T} \int_{\Omega}\left(R+\tau_{\Delta t} \eta_{N}\right)^{2}\left(\partial_{t} \tilde{\mathbf{u}}_{N} \cdot \mathbf{q}_{N}+\frac{1}{2}\left(\tau_{\Delta t} \mathbf{u}_{N}-\mathbf{w}_{N}\right) \cdot \nabla^{\tau_{\Delta t} \eta_{N}} \mathbf{u}_{N} \cdot \mathbf{q}_{N}\right. \\
\left.-\frac{1}{2}\left(\tau_{\Delta t} \mathbf{u}_{N}-\mathbf{w}_{N}\right) \cdot \nabla^{\tau_{\Delta t} \eta_{N}} \mathbf{q}_{N} \cdot \mathbf{u}_{N}\right)+\rho_{f} \int_{0}^{T}\left(R+\frac{1}{2}\left(\tau_{\Delta t} \eta_{N}+\eta_{N}\right)\right) \int_{\Omega} v_{N}^{*} \mathbf{u}_{N} \cdot \mathbf{q}_{N} \\
+\int_{0}^{T} \int_{\Omega}\left(R+\tau_{\Delta t} \eta_{N}\right)^{2} 2 \mu_{F} \mathbf{D}^{\tau_{\Delta t} \eta_{N}}\left(\mathbf{u}_{\mathbf{N}}\right): \mathbf{D}^{\tau_{\Delta t} \eta_{N}}\left(\mathbf{q}_{N}\right)+R \rho_{K} h \int_{0}^{T} \int_{\omega} \partial_{t} \tilde{v}_{N} \psi \\
+\frac{R h}{2} \int_{0}^{T} \int_{\omega} Q \boldsymbol{G}(\eta): \frac{1}{2}\left(\mathbf{G}^{\prime}\left(\eta_{N}\right)+\mathbf{G}^{\prime}\left(\tau_{\Delta t} \eta_{N}\right)\right) \psi+R \varepsilon \int_{0}^{T} \int_{\omega} \Delta \eta \Delta \psi \\
=R\left(\int_{0}^{T} P_{\text {in }}^{N} \int_{\Gamma_{\text {in }}}\left(q_{z}\right)_{\mid z=0}-\int_{0}^{T} P_{\text {out }}^{N} \int_{\Gamma_{\text {out }}}\left(q_{z}\right)_{\mid z=L}\right),
\end{gathered}
$$

with

$$
\begin{gathered}
\nabla^{\tau_{\Delta t} \eta} \cdot \mathbf{u}_{N}=0, \quad v_{N}=\left(\left(u_{r}\right)_{N}\right)_{\mid \Gamma}, \\
\mathbf{u}_{N}(0, .)=\mathbf{u}_{0}, \eta(0, .)_{N}=\eta_{0}, v_{N}(0, .)=v_{0} .
\end{gathered}
$$

Here $\tilde{\mathbf{u}}_{N}$ and $\tilde{v}_{N}$ are the piecewise linear functions defined in (6.7), $\tau_{\Delta t}$ is the shift in time by $\Delta t$ to the left, defined in (6.3), $\nabla^{\tau_{\Delta t} \eta_{N}}$ is the transformed gradient via the ALE mapping $A_{\tau_{\Delta t} \eta_{N}}$, defined in (4.5), and $v_{N}^{*}, \mathbf{u}_{N}, v_{N}$ and $\eta_{N}$ are defined in (6.1).

Now we can use the convergence results from Lemma 11 and Theorem 13 to pass to the limit in (7.1) and (7.2) in the analogous way as in [36]. The only difference is the nonlinear term

$$
Q \mathbf{G}\left(\eta_{N}\right): \mathbf{G}^{\prime}\left(\eta_{N}, \tau_{\Delta t} \eta_{N}\right) \psi=Q \mathbf{G}\left(\eta_{N}\right): \frac{1}{2}\left(\mathbf{G}^{\prime}\left(\eta_{N}\right)+\mathbf{G}^{\prime}\left(\tau_{\Delta t} \eta_{N}\right)\right) \psi .
$$

This terms consists of the sums and products of the functions $\eta_{N}, \tau_{\Delta t} \eta_{N}$ and their first order derivatives. Form the strong convergence results presented in Theorem 13 we have:

$$
\eta_{N} \rightarrow \eta \text { and } \tau_{\Delta t} \eta_{N} \rightarrow \eta \text { in } L^{\infty}\left(0, T ; W^{1,4}(\omega)\right) .
$$

Therefore we can directly pass to the limit in the nonlinear term to obtain that the final result, namely, that the limit of the sequence of approximate functions defined by the operator splitting scheme described in Section 5 satisfies the weak formulation of the coupled FSI problem (4.19), and defines a weak solution of the coupled FSI problem. More precisely, the following theorem holds true:

THEOREM 15 Let $\varrho_{f}$ (fluid density), $\varrho_{K}$ (structure density), $\mu_{F}$ (fluid viscosity), $h$ (structure thickness), $\mu$ and $\lambda$ (Lamé constants), all be strictly positive. Suppose that the initial data $v_{0} \in$ $L^{2}(\omega), \mathbf{u}_{0} \in L^{2}\left(\Omega_{\eta_{0}}\right)$, and $\eta_{0} \in H_{0}^{2}(\omega)$ are such that $\left(R+\eta_{0}(z)\right)>0, z \in[0, L]$. Furthermore, let $P_{\text {in }}, P_{\text {out }} \in L_{\text {loc }}^{2}(0, \infty)$.

Then there exist a time $T>0$ and a weak solution of $(\mathbf{u}, \eta)$ of Problem 2 on $(0, T)$ in the sense of Definition 2, which satisfy the following energy estimate:

$$
E(t)+\int_{0}^{t} D(\tau) d \tau \leqslant E_{0}+C\left(\left\|P_{\text {in }}\right\|_{L^{2}(0, t)}^{2}+\left\|P_{\text {out }}\right\|_{L^{2}(0, t)}^{2}\right), \quad t \in[0, T],
$$

where $C$ depends only on the coefficients in the problem, $E_{0}$ is the kinetic energy of initial data, 
and $E(t)$ and $D(t)$ are given by

$$
\begin{aligned}
& E(t)=\frac{\rho_{f}}{2}\|\mathbf{u}\|_{L^{2}\left(\Omega_{\eta}(t)\right)}^{2}+\frac{\rho_{F} h}{2}\left\|\partial_{t} \eta\right\|_{L^{2}(\omega)}^{2}+\frac{1}{2}\left(\frac{1}{2}\|\eta\|_{\gamma}^{4}+\varepsilon\|\Delta \eta\|\right), \\
& D(t)=\mu_{F}\|\mathbf{D}(\mathbf{u})\|_{L^{2}(\Omega)}^{2} .
\end{aligned}
$$

Furthermore, one of the following is true: either

1. $T=\infty$, or

2. $\lim _{t \rightarrow T} \min _{z \in[0, L]}(R+\eta(z))=0$.

Notice that the last assertion of the theorem states that our existence result is "global" in time in the sense that the solution exists until the walls of the cylinder touch each other. The proof of this argument is the same as in [36] and [6, p. 397-398] and we omit it here.

This theorem shows the existence of a weak solution to Problem 2, which is the original FSI problem mapped onto a fixed domain $\Omega$. By mapping Problem 2 back onto the physical domain via the ALE mapping $A_{\eta}$, where $\eta$ is the limiting structure displacement, this theorem implies the existence of a weak solution of the FSI problem listed under Problem 1.

Acknowledgements. The authors would like to thank Josip Tambača for his help and notes on the Koiter shell model, and Maroje Marohnić for discussions. The authors would also like to acknowledge research support by the Croatian Science Foundation under grant number 9477, and by NSF under DMS-1311709 (Muha), and the support by NSF under grants DMS-1263572, DMS1318763, DMS-1311709, DMS-1262385 and DMS-1109189 (Čanić).

\section{REFERENCES}

1. Barbu, V., Grujić, Z., LASiecka, I. \& Tuffaha, A., Existence of the energy-level weak solutions for a nonlinear fluid-structure interaction model. In Fluids and waves, volume 440 of Contemp. Math., 55-82. Amer. Math. Soc., Providence, RI, 2007. Zbl1297. 35234 MR2359449

2. Barbu, V., Grujić, Z., LAsiecka, I. \& Tuffaha, A., Smoothness of weak solutions to a nonlinear fluid-structure interaction model. Indiana Univ. Math. J. 57 (2008), 1173-1207. Zbl1147.74016 MR2429089

3. BEIRÃO DA VEIGA, H., On the existence of strong solutions to a coupled fluid-structure evolution problem. J. Math. Fluid Mech. 6 (2004), 21-52. Zbl1068.35087 MR2027753

4. Bukač, M., Yotov, I. \& Zunino, P., An operator splitting approach for the interaction between a fluid and a multilayered poroelastic structure. Numer. Methods Partial Differential Equations 31 (2015), 1054-1100. Zb106497871 MR3343599

5. BUKaČ, M., ČAniĆ, S., GLOWINSKI, R., TAMbaČA, J. \& QUAINI, A., Fluid-structure interaction in blood flow capturing non-zero longitudinal structure displacement. Journal of Computational Physics 235 (2013), 515-541. MR3017610

6. Chambolle, A., Desjardins, B., Esteban, M. J. \& Grandmont, C., Existence of weak solutions for the unsteady interaction of a viscous fluid with an elastic plate. J. Math. Fluid Mech. 7 (2005), 368-404. Zbl1080.74024 MR2166981

7. Cheng, C. H. A., Coutand, D. \& Shkoller, S., Navier-Stokes equations interacting with a nonlinear elastic biofluid shell. SIAM J. Math. Anal. 39 (2007), 742-800 (electronic). Zb11138. 74022 MR2349865

8. Cheng, C. H. A. \& ShKoller, S., The interaction of the 3D Navier-Stokes equations with a moving nonlinear Koiter elastic shell. SIAM J. Math. Anal. 42 (2010), 1094-1155. Zbl 05876592 MR2644917 
9. I. Chueshov. Interaction of an elastic plate with a linearized inviscid incompressible fluid. Comm. Pure Appl. Analys 13 (2014), 1759-1778. Zbl1305.74029 MR3230411

10. Ciarlet, P. G., Un modèle bi-dimensionnel non linéaire de coque analogue à celui de W. T. Koiter. $C$. R. Acad. Sci. Paris Sér. I Math., 331 (2000), 405-410. Zb10994.74044 MR1784923

11. Ciarlet, P. G. \& Roquefort, A., Justification d'un modèle bi-dimensionnel non linéaire de coque analogue à celui de W. T. Koiter. C. R. Acad. Sci. Paris Sér. I Math. 331 (2000), 411-416. Zbl0994. 74045 MR1784924

12. Conca, C., Murat, F. \& Pironneau, O., The Stokes and Navier-Stokes equations with boundary conditions involving the pressure. Japan. J. Math. (N.S.) 20 (1994), 279-318. Zbl0826.35093 MR1308419

13. Coutand, D. \& Shkoller, S., Motion of an elastic solid inside an incompressible viscous fluid. Arch. Ration. Mech. Anal. 176 (2005), 25-102. Zbl1064. 74057 MR2185858

14. Coutand, D. \& ShKoller, S., The interaction between quasilinear elastodynamics and the NavierStokes equations. Arch. Ration. Mech. Anal. 179 (2006), 303-352. Zbl1138. 74325 MR2208319

15. DonÉA, J., A Taylor-Galerkin method for convective transport problems. In Numerical methods in laminar and turbulent flow (Seattle, Wash., 1983), pages 941-950. Pineridge, Swansea, 1983. Zb10553. 76082 MR0830474

16. Du, Q. , Gunzburger, M. D., Hou, L. S. \& Lee, J., Analysis of a linear fluid-structure interaction problem. Discrete Contin. Dyn. Syst., 9 (2003), 633-650. Zbl1039. 35076 MR1974530

17. Gilbarg, D. \& Trudinger, N. S., Elliptic Partial Differential Equations of Second Order (Second Edition). Springer-Verlag, Heidelberg, 1983. Zb10562.35001 MR0737190

18. Glowinski, R., Finite element methods for incompressible viscous flow, in: P.G.Ciarlet, J.-L.Lions (Eds), Handbook of numerical analysis, volume 9. North-Holland, Amsterdam, 2003. Zbl1040.76001 MR2009826

19. GRAndmont, C., Existence of weak solutions for the unsteady interaction of a viscous fluid with an elastic plate. SIAM J. Math. Anal. 40 (2008), 716-737. Zbl1158.74016 MR2438783

20. GRISVARD, P., Singularities in boundary value problems, volume 22 of Recherches en Mathématiques Appliquées [Research in Applied Mathematics]. Masson, Paris, 1992. Zb10766.35001 MR1173209

21. Guidoboni, G., Glowinski, R., CAVAllini, N. \& ČAniĆ, S., Stable loosely-coupled-type algorithm for fluid-structure interaction in blood flow. Journal of Computational Physics 228 (2009), 6916-6937. Zbl1261. 76056 MR2567876

22. Guidoboni, G., Guidorzi, M. \& Padula, M., Continuous dependence on initial data in fluid-structure motions. J. Math. Fluid Mech. 14 (2012), 1-32. Zbl1294. 35099 MR2891187

23. Hundertmark-ZaušKová, A., LuKÁčová-Medvid̉ová, M. \& RusnÁKovÁ, G., Fluid-structure interaction for shear-dependent non-Newtonian fluids. In Topics in mathematical modeling and analysis, volume 7 of Jindřich Nečas Cent. Math. Model. Lect. Notes, pages 109-158. Matfyzpress, Prague, 2012. MR2962823

24. Ignatova, M., KukavicA, I., LasieckA, I. \& Tuffaha, A., On well-posedness for a free boundary fluid-structure model. J. Math. Phys. 53 (2012), 115624. Zbl06245454 MR3026569

25. Ignatova, M., Kukavica, I., Lasiecka, I. \& Tuffaha, A., On well-posedness and small data global existence for an interface damped free boundary fluid-structure model. Nonlinearity 27 (2014), 467. Zbl1286. 35272 MR3168261

26. KoIter, W. T., On the foundations of the linear theory of thin elastic shells. I, II. Nederl. Akad. Wetensch. Proc. Ser. B 73 (1970), 169-182; ibid., 73 (1970), 183-195. Zbl0213. 27002 MR0280050

27. Kukavica, I. \& Tuffaha, A., Solutions to a fluid-structure interaction free boundary problem. DCDS-A 32 (2012), 1355-1389. Zbl1234. 35320 MR2851902

28. Kukavica, I. \& Tuffaha, A., Solutions to a free boundary problem of fluid-structure interaction. Indiana Univ. Math. J. 61 (2012), 1817-1859. Zb11280. 35094 MR3119602 
29. Kukavica, I. \& Tuffaha, A., Well-posedness for the compressible Navier-Stokes-Lamé system with a free interface. Nonlinearity 25 (2012), 3111-3137. Zbl1256.35213 MR2991431

30. Kukavica, I., Tuffaha, A. \& Ziane, M., Strong solutions for a fluid structure interaction system. Adv. Differential Equations 15 (2010), 231-254. Zbl1191. 35206 MR2588449

31. LENGELER, D. \& RǓŽIČKA, M., Weak solutions for an incompressible newtonian fluid interacting with a koiter type shell. Archive for Rational Mechanics and Analysis 211 (2014), 205-255. Zbl1293.35211 MR3147436

32. LEQUEURRE, J., Existence of strong solutions to a fluid-structure system. SIAM J. Math. Anal. 43 (2011), 389-410. Zbl1232.35118 MR2765696

33. LEQUEURRE, J., Existence of strong solutions for a system coupling the Navier-Stokes equations and a damped wave equation. J. Math. Fluid Mech. 15 (2013), 249-271. Zbl06180450 MR3061763

34. LukÁčová-Medvid’ová, M., Rusnáková, G. \& Hundertmark-ZaušKová, A., Kinematic splitting algorithm for fluid-structure interaction in hemodynamics. Computer Methods in Applied Mechanics and Engineering 265 (2013), 83-106. Zbl1286 . 76176 MR3093603

35. Muna, B., A note on the trace theorem for domains which are locally subgraph of a Hölder continuous function. Netw. Heterog. Media 9 (2014), 191-196. Zbl1305.74031 MR3195351

36. Muha, B. \& ČAnić, S., Existence of a Weak Solution to a Nonlinear Fluid-Structure Interaction Problem Modeling the Flow of an Incompressible, Viscous Fluid in a Cylinder with Deformable Walls. Arch. Ration. Mech. Anal. 207 (2013), 919-968. Zbl1260.35148 MR3017292

37. MuHA, B. \& ČAnIĆ, S., Existence of a solution to a fluid-multi-layered-structure interaction problem. J. Differential Equations 256 (2014), 658-706. Zb106447075 MR3121710

38. MunA, B. \& ČANIĆ, S., A nonlinear, 3D fluid-structure interaction problem driven by the time-dependent dynamic pressure data: a constructive existence proof. Communications in Information and Systems $\mathbf{1 3}$ (2013), 357-397. Zbl06402861 MR3226949

39. Quarteroni, A. Tuveri, M. \& Veneziani, A., Computational vascular fluid dynamics: problems, models and methods. Computing and Visualization in Science 2 (2000), 163-197. Zbl1096 76042

40. RAYMOND, J.-P. \& VANNINATHAN, M., A fluid-structure model coupling the navier-stokes equations and the lamé system. J. Math. Pures Appl. 9102 (2014), 546-596. Zbl1303.35060 MR3249722

41. Simon, J., Compact sets in the space $L^{p}(0, T ; B)$. Ann. Mat. Pura Appl. 4146 (1987), 65-96. Zb10629. 46031 MR0916688

42. Smart, R., Fixed Points Theorems. Cambridge University Press, New York, 1980. Zb10427. 47036

43. Temam, R., Navier-Stokes equations. Theory and numerical analysis. North-Holland Publishing Co., Amsterdam, 1977. Studies in Mathematics and its Applications, Vol. 2.

44. ČAnIĆ, S., MUHA, B. \& BUKAČ, M., Stability of the kinematically coupled $\beta$-scheme for fluid-structure interaction problems in hemodynamics. Int J Numer Anal Model 12 (2015), 54-80. MR3286456 